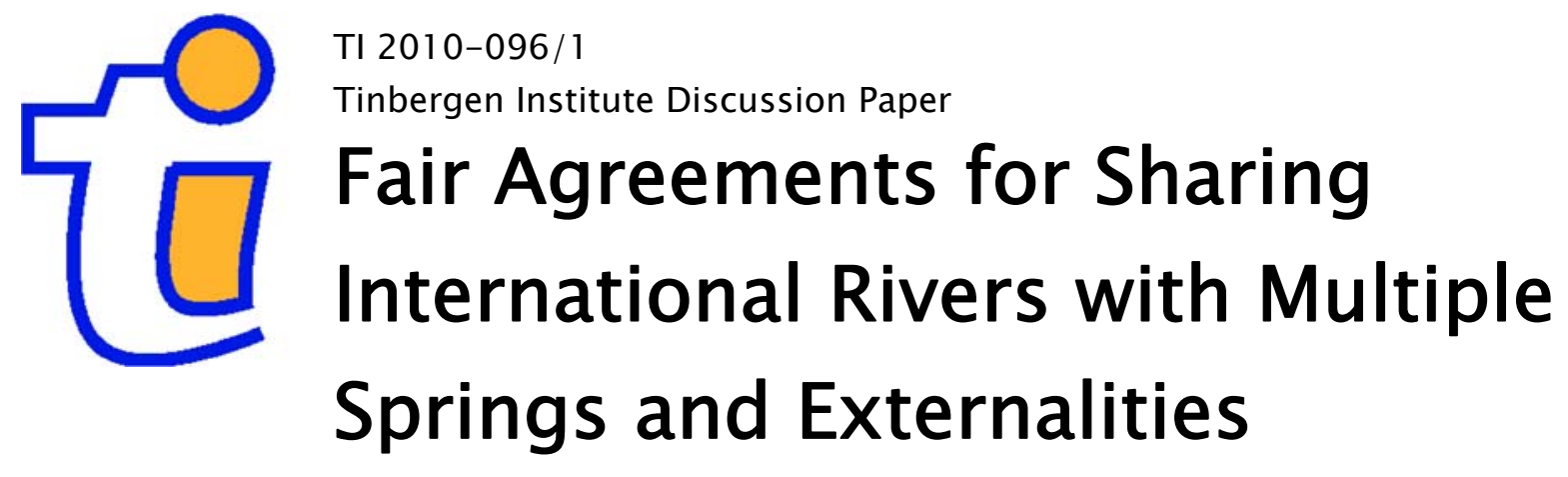

René van den Brink

Gerard van der Laan

Nigel Moes

VU University Amsterdam, and Tinbergen Institute. 


\section{Tinbergen Institute}

The Tinbergen Institute is the institute for economic research of the Erasmus Universiteit Rotterdam, Universiteit van Amsterdam, and Vrije Universiteit Amsterdam.

Tinbergen Institute Amsterdam

Roetersstraat 31

1018 WB Amsterdam

The Netherlands

Tel.: +31(0)205513500

Fax: $+31(0) 205513555$

Tinbergen Institute Rotterdam

Burg. Oudlaan 50

3062 PA Rotterdam

The Netherlands

Tel.: + $31(0) 104088900$

Fax: $+31(0) 104089031$

Most TI discussion papers can be downloaded at http://www.tinbergen.nl. 


\title{
Fair agreements for sharing international rivers with multiple springs and externalities ${ }^{1}$
}

\author{
René van den Brink ${ }^{2}$ Gerard van der Laan ${ }^{3}$ Nigel Moes ${ }^{4}$
}

September 16, 2010

\footnotetext{
${ }^{1}$ This research is financially supported by Netherlands Organization for Scientific Research, NWO grant 400-07-159.

${ }^{2}$ J.R. van den Brink, Department of Econometrics and Tinbergen Institute, VU University Amsterdam, De Boelelaan 1105, 1081 HV Amsterdam, The Netherlands. E-mail: jrbrink@feweb.vu.nl

${ }^{3}$ G. van der Laan, Department of Econometrics and Tinbergen Institute, VU University Amsterdam, De Boelelaan 1105, 1081 HV Amsterdam, The Netherlands. E-mail: glaan@feweb.vu.nl

${ }^{4}$ N. Moes, Department of Econometrics and Tinbergen Institute, VU University Amsterdam, De Boelelaan 1105, 1081 HV Amsterdam, The Netherlands. E-mail: nmoes@feweb.vu.nl
} 


\begin{abstract}
In this paper we consider the problem of sharing water from a river among a group of agents (countries, cities, firms) located along the river. The benefit of each agent depends on the amount of water consumed by the agent. An allocation of the water among the agents is efficient when it maximizes the total benefits. To sustain an efficient water allocation, the agents can compensate each other by paying monetary transfers. Every water allocation and transfer schedule yields a welfare distribution, where the utility of an agent is equal to its benefit from the water consumption plus its monetary transfer (which can be negative). The problem of finding a fair welfare distribution can be modelled by a cooperative game.

For a river with one spring and increasing benefit functions, Ambec and Sprumont (2002) propose the downstream incremental solution as the unique welfare distribution that is core-stable and satisfies the condition that no agent gets a utility payoff above its aspiration level. Ambec and Ehlers (2008) generalized the Ambec and Sprumont river game to river situations with satiable agents, i.e., the benefit function is decreasing beyond some satiation point. In such situations externalities appear, yielding a cooperative game in partition function form. In this paper we consider river situations with satiable agents and with multiple springs. For this type of river systems we propose the class of so-called weighted hierarchical solutions as the class of solutions satisfying several principles to be taken into account for solving water disputes. When every agent has an increasing benefit function (no externalities) then every weighted hierarchical solution is core-stable. In case of satiation points, it appears that every weighted hierarchical solution is independent of the externalities.
\end{abstract}

Keywords: Water allocation, river game, externality, core, hierarchical outcome.

JEL code: C71, D62, H23 


\section{Introduction}

The aim of this paper is to introduce and analyze rules for a fair distribution of welfare resulting from allocating the water flow of a river among the agents (countries, cities, firms) along the river. We consider the case of an international river, where the agents are countries located from upstream to downstream along the river, and water flows possible from the inland into the river on every agent's territory. ${ }^{1}$ Since water flows from upstream to downstream, water inflow at the territory of downstream agents can not be consumed by upstream agents. This is in contrast to a transboundary river where the river forms a common border and all countries have equal access to the water. A welfare maximizing allocation of the water flow among the agents typically requires that some agents do not consume the total inflow on their own territory, but that upstream agents let pass some of the water flow to their downstream agents, who derive higher welfare from the water. However, since every agent benefits from the consumption of water, giving up water is regarded as unacceptable. Therefore monetary compensation schemes are required in order to make a welfare maximizing allocation implementable. International law states that the nations involved should mutually agree on sharing the river through negotiations, but it is left in the middle to what extend unilateral decisions can be made in the absence of agreement. Moreover, such negotiations are often deadlocked, because almost all governments in water stressed regions became aware of the water issues after having experienced serious shortages. Unless politics either deepens or broadens the water agenda, as in e.g. Bennett, Ragland and Yolles (1998), the situation is most likely to stay put or might even deteriorate ending in conflict.

Coalition formation, the division of gains within coalitions, unilateral decisions prior to the negotiations and incentive compatibility to sustain an agreement, belong traditionally to the realm of game theory. This is also recognized by global institutions involved in river management such as the World Bank, see for instance Carraro, Machiori and Sgobbi (2005a,b). Many researchers in economics and game theory have addressed the water issue, see for instance Dinar, Ratner and Yaron (1992) and Dinar et al. (2005) for extensive surveys. A lot of this research focuses on the problem of allocating water in which a common resource has to be shared by several users, for instance allocating water in case of transboundary rivers or in case of common groundwater aquifers. In contrast to such common pool situations, a feature peculiar to international rivers is the one-directionality of the water flow, imposing in some sense dominance of upstream agents over downstream agents. International water law, as laid down in the Helsinki Rules of 1966 and the UN Convention on the Law of the Non-Navigational Uses of International Watercourses of

\footnotetext{
${ }^{1}$ The results also apply when the agents are cities or firms along the river within one country.
} 
1997, does neither recognize claims by upstream countries on the water caught on its territory, nor downstream nations claims of historical rights, which makes the application of negotiation theory even more difficult. The international river problem is studied in for instance Kilgour and Dinar (1995, 2001), Bennett, Ragland and Yolles (1998), Ambec and Sprumont (2002), Supalla et al. (2002) and Ambec and Ehlers (2008).

To address the problem of a fair welfare distribution, Ambec and Sprumont (2002) model the international river problem as a cooperative game on the set of agents (countries). For a river with one spring and one sink, i.e., the agents are successively located from the most upstream agent at the spring of the river to the most downstream agent at the sink, they propose and axiomatize the so-called downstream incremental distribution. This is the welfare distribution that assigns all gains of cooperation between agents in a coalition to the most downstream agent, subject to the core stability constraints of all upstream coalitions. For a two agent situation this means the following. To maximize total welfare, typically part of the water inflow on the territory of the upstream agent goes to the downstream agent. The downstream agent pays an amount of money just enough to compensate the upstream agent for the loss of utility caused by transferring part of his water inflow to the downstream agent. So, the downstream incremental solution makes the upstream agent indifferent between the optimal allocation of water with the monetary compensation or using all his water by himself. The downstream agent receives all the surplus of cooperation. In van den Brink, van der Laan and Vasil'ev (2007) it is argued that the downstream incremental distribution ignores the dominance, caused by the one-directionality of the water flow, of the upstream agents over the downstream agents. For example, in the two agent situation mentioned above, the upstream agent can claim all gains from cooperation by playing the ultimatum game, refusing cooperation with the downstream agent when the latter agent does not agree to let all gains of the cooperation to the upstream agent and thus making the downstream agent indifferent between cooperation or not. In contrast to this 'negotiation power' of the upstream agent, the downstream incremental distribution gives all gains from cooperation to the downstream agent. In van den Brink, van der Laan and Vasil'ev (2007) it has been shown that replacing one of the characterizing properties of the downstream incremental distribution by some other property, yields the alternative upstream incremental welfare distribution. This distribution assigns all gains of cooperation between agents in a coalition to the most upstream agent, subject to the core stability constraints of all downstream coalitions. Within a different solution concept, the same result is obtained in Herings, van der Laan and Talman (2007). In both papers it is argued that the upstream incremental distribution is in accordance to the dominance of the upstream agents because the one-directionality of the water flow. 
In this paper we want to derive, within the framework of a cooperative game, fair welfare distributions for international rivers with multiple springs (i.e. there are side rivers merging into one mainstream), by taking into account well-known principles in international water allocation problems. In Kilgour and Dinar (1995) several principles have been discussed to prevent or resolve disputes on water allocation within an international water basin. Since there is no binding international law governing the allocation of water in international rivers, these principles, in combination with the 1966 Helsinki Document (see Kilgour and Dinar), are the only guidelines that are available in determining a fair distribution of the welfare resulting from the water allocation among the agents. ${ }^{2}$

According to the 1966 Helsinki Document two considerations that are to be included when distributing the welfare from allocating the water are 'efficiency of the water use' and 'Pareto optimality'. Efficiency of water use and Pareto optimality imply that (i) every coalition of agents distributes the water over its members in such a way that no water is wasted and the water ends up at the agent(s) that derive(s) the greatest (marginal) utility from it, and (ii) the monetary transfers are budget balanced, i.e., the sum of all (positive and negative) payments over all agents is equal to zero. To attain the first condition the marginal utilities are equalized as much as possible given the constraints arising from the one-directionality of the water flow. The other principles discussed by Kilgour and Dinar (1995) concern the 'water rights' assigned to the different agents (countries) along the river. The principle of Absolute Territorial Sovereignty (ATS), or Harmon doctrine, states that an agent has absolute sovereignty over the area of any river basin on its territory. This principle clearly favors upstream agents in that it allows agents to use any water that flows into the river on their territory without taking into account what consequences this might have for the countries downstream to it. Within the cooperative game theoretical framework of this paper this principle results in the requirement that the welfare distribution should be core-stable. That means that every coalition of connected agents (i.e. for every two agents in the coalition, every agent that is passed when going along the river from the first to the second agent is also in the coalition) can secure themselves the welfare level that can be reached by allocating the water that they control optimally amongst themselves, given the constraints from the one-directionality of the flow and thus the monetary transfers should be at least big enough to compensate them for any loss of welfare due to allocating water from their territory to agents outside the coalition.

The second doctrine listed in Kilgour and Dinar (1995) is the principle of Territorial Integration of all Basin States (TIBS). This principle favors downstream states, to which it accords "equal" use, without regard to their contribution to the flow. It does not consider

\footnotetext{
${ }^{2}$ These principles can, of course, also be used when the agents in the model are not countries but, for instance, firms or cities.
} 
any country the legal owner of the water, but instead it states that the water belongs to all the countries, no matter where it enters the river. Of course, also when applying this principle we do have to take into account the one-directionality of the water flow and thus the assignment of water rights has to be compatible with the actual territorial inflows. Ambec and Sprumont (2002) apply an extreme case of the TIBS principle, called Unlimited Territorial Integrity (UTI). For the international river with a single spring, they conclude from the UTI principle that the welfare distribution should be such that no coalition should get a total utility above its so-called aspiration level, defined as the maximum worth the coalition can attain by an optimal allocation among its members of the total water inflow from the spring to the most downstream member of the coalition. Combining this aspiration level requirement with the core-stability requirement from the ATS principle yields the downstream incremental welfare distribution.

In this paper we allow for more flexible interpretations of the TIBS principle, including the above UTI principle as an extreme case. The general TIBS principle states that, given some agent, this agent and all its downstream agents are entitled to receive 'a share' in the water inflow at that agent. Since in this paper water and utility are interchangeable (consumption of water yields utility), to say that a certain country has the right to a certain share in the water flow comes down to saying that a country has the right to a certain share in the welfare resulting from the water flow. The TIBS principle therefore implies that each agent has the right to a specific amount of utility (corresponding to the share of water that it has the right to according to the principle). Suppose that, for one reason or another, the agents along a river with multiple springs are cooperating in two separate coalitions as follows. For some agent, say $i$, one of the coalitions consists of agent $i$ and all its upstream agents, and the other coalition is its complement, i.e., consists of all other agents. For instance this happens when agent $i$ is not willing to cooperate with its unique downstream neighbor. The question that then can be asked is: how should the gain in welfare (utility) that is created when the two coalitions join together into one coalition of all agents, be divided among the agents? Evidently, we can ask this question for every single agent $i$ (except the unique most downstream agent). The TIBS principle provides us with an answer. First, notice that according to the TIBS principle every agent is entitled to receive a certain share of the water inflow, but that it does not specify these shares and even does not require the shares to be equal. Now, let there be for each agent a nonnegative number, its weight, with sum over all agents equal to one, which corresponds to the share of the agent when all agents cooperate together. Then we interpret the TIBS principle by requiring that for each agent $i$ the gain in welfare that is created by merging $i$ 's upstream coalition and its complement, should be divided among the two coalitions proportional to the sum of the weights of the agents in these two coalitions. We will show that for every 
specific vector of weights this requirement, together with efficiency, characterizes a particular welfare distribution resulting from a welfare maximizing water allocation. The extreme case where the most downstream agent has weight equal to one yields the downstream incremental welfare distribution, the special case that all weights are taken to be equal yields a welfare distribution that can be seen as taking the average of so-called hierarchical outcomes as introduced by Demange (2004), see also Herings, van der Laan and Talman (2008).

In Ambec and Sprumont (2002) it is assumed that the utility that an agent derives from water consumption is strictly increasing in the amount of water. Under this assumption only coalitions of consecutive agents will form. When for instance a coalition consists of an upstream consecutive part and a downstream consecutive part with in-between some agents outside the coalition, then every water flow sent from the upstream part to the downstream part would immediately be taken by the in-between agents. Ambec and Ehlers (2008) allow for satiable agents. For such an agent the utility is increasing up to a certain amount of water, but decreasing beyond that amount. The existence of satiation points has serious consequences, because now also coalitions of non-consecutive agents might form. When every in-between outside agent has a satiation point, then it might be profitable for the upstream part of a coalition to transfer water to the downstream part. Although some of this flow might be taken by the in-between agents, these agents will only take water up to their satiation points. When the flow is big enough, cooperation between the upstream part and the downstream part could be profitable. Now, consider some coalition of consecutive agents. Without satiated agents, such a coalition of consecutive agents obtains its maximum welfare by allocating optimally the water inflow on their own territory among themselves. This maximum welfare does not depend on the behavior of the agents upstream and downstream of the coalition, because the upstream agents will never pass water to the downstream agents. However, in case of satiation, this might happen and when it happens, the agents in-between might take part of this water flow up to their satiation points. Therefore the welfare of a coalition of consecutive agents might be affected positively when the agents upstream of the coalition are going to cooperate with the agents downstream of the coalition, i.e., a coalition might experience positive externalities from cooperation between the upstream and downstream agents outside the coalition ${ }^{3}$.

Ambec and Ehlers (2008) generalizes the model of Ambec and Sprumont for externalities, but still consider rivers with one spring and one sink. In this paper we consider river

\footnotetext{
${ }^{3}$ This type of externality is consistent with the definition given by e.g. Meade (1973): 'An external economy (diseconomy) is an event which confers an appreciable benefit (inflicts an appreciable damage) on some person or persons who were not fully consenting parties in reaching the decision or decisions which led directly or indirectly to the event in question.'
} 
situations with multiple springs and allowing for externalities. In general, situations in which the formation of a coalition yields externalities on the agents outside the coalition can be modeled by a cooperative game in partition function form, as proposed in Thrall and Lucas (1963). ${ }^{4}$ Applying the efficiency and TIBS principles mentioned above to river games in partition function form, we will see that these axioms imply an externality-free solution, i.e., this solution can be obtained by associating a standard cooperative game to the game in partition function form.

The paper is organized as follows. Section 2 contains preliminaries on cooperative games, Section 3 introduces the cooperative game model for international rivers with one spring and one sink and then generalizes to a cooperative game model for rivers with multiple springs and to games in partition function form for rivers with multiple springs and satiated agents. In Section 4 we discuss several principles for allocating water in international rivers with multiple springs, but without satiated agents, and specify these principles into axioms for solutions in our game model. We characterize the solutions that are determined by these principles. In Section 5 we discuss the implications of this for the special case of a river with only one spring, and in Section 6 we discuss the case with satiated agents. Finally, Section 7 contains concluding remarks.

\section{Preliminaries on cooperative games}

A cooperative game with transferable utility in characteristic function form, or simply a TU-game, is a pair $(N, v)$, where $N=\{1, \ldots, n\}$ is a finite set of $n$ players, and $v: 2^{N} \rightarrow \mathbb{R}$ is a characteristic function on $N$ such that $v(\emptyset)=0$. For any coalition $S \subseteq N, v(S)$ is the worth of coalition $S$, i.e., the members of coalition $S$ can obtain a total payoff of $v(S)$ by agreeing to cooperate. When we take the player set $N$ to be fixed, we represent a TU-game by its characteristic function $v$ and we denote the collection of all TU-games on $N$ by $\mathcal{G}^{N}$. A game $v \in \mathcal{G}^{N}$ is superadditive if $v(S)+v(T) \leq v(S \cup T)$ for any pair of subsets $S, T \subseteq N$ such that $S \cap T=\emptyset$. Further, a game $v \in \mathcal{G}^{N}$ is convex if $v(S)+v(T) \leq v(S \cup T)+v(S \cap T)$ for all $S, T \subseteq N$.

For a collection of games $\mathcal{G} \subseteq \mathcal{G}^{N}$, a set-valued solution $F$ on $\mathcal{G}$ assigns a set $F(v) \subset \mathbb{R}^{n}$ of payoff vectors to every TU-game $v \in \mathcal{G}$. The most applied set-valued solution on $\mathcal{G}^{N}$ is the Core (Gillies, 1953) which assigns to every TU-game $v \in \mathcal{G}^{N}$ the set

\footnotetext{
${ }^{4}$ Despite its early introduction, the partition function form turned out to be a methodological tough problem, and only during the last decade breakthroughs are reported, see e.g. Funaki and Yamato (1999), Ray and Vohra (1999), Albizuri, Arin and Rubio (2005), Gomez (2005), Gomez and Jehiel (2005), De Clippel and Serrano (2008) and Albizuri (2010).
} 
given by

$$
\operatorname{Core}(v)=\left\{x \in \mathbb{R}^{n} \mid \sum_{i \in N} x_{i}=v(N) \text { and } \sum_{i \in S} x_{i} \geq v(S) \text { for all } S \subset N\right\},
$$

i.e., the core is the set of all efficient payoff vectors that are stable in the sense that no coalition $S \subset N$ can do better by deviating from the grand coalition $N$ and realising its own worth $v(S)$. The core of a game is nonempty if and only if $v$ is balanced, see e.g. Bondareva (1963) or Shapley (1967). Since every convex game is balanced, it follows that the core is nonempty for every convex game.

A single-valued solution $f$ on $\mathcal{G} \subseteq \mathcal{G}^{N}$ assigns precisely one payoff vector $f(v) \in \mathbb{R}^{n}$ to every $v \in \mathcal{G}$. The most applied single-valued solution on $\mathcal{G}^{N}$ is the Shapley value which is defined as the average of all marginal vectors over all permutations of the players. For a permutation $\pi: N \rightarrow N$, assigning rank number $\pi(i) \in N$ to any player $i \in N$, we define $\pi^{i}=\{j \in N \mid \pi(j) \leq \pi(i)\}$, i.e., $\pi^{i}$ is the set of all players with rank number at most equal to the rank number of $i$, including $i$ itself. Then the marginal vector $m^{\pi}(v) \in \mathbb{R}^{n}$ of game $v$ and permutation $\pi$ is given by $m_{i}^{\pi}(v)=v\left(\pi^{i}\right)-v\left(\pi^{i} \backslash\{i\}\right), i \in N$. Denoting by $\Pi(N)$ the collection of all permutations on player set $N$, the Shapley value (Shapley (1953)) $S h: \mathcal{G}^{N} \rightarrow \mathbb{R}^{n}$ is given by

$$
S h_{i}(v)=\frac{1}{n !} \sum_{\pi \in \Pi(N)} m_{i}^{\pi}(v) \text { for all } i \in N .
$$

For convex games each marginal vector lies in the core of the game, and by convexity of the core also the Shapley value and any other convex combination of marginal vectors belongs to the core.

In a TU-game the worth of a coalition does not depend on the activities of players outside the coalition, and thus does not take account of externalities on the worth of a coalition resulting from the formation of coalitions of agents outside the coalition. Situations in which the worth of a coalition $S \subset N$ depends on the coalition formation outside $S$ can be modeled by a cooperative game with transferable utility in partition function form, or shortly a PFF-game. A partition $\mathcal{P}=\left\{S_{1}, \ldots, S_{k}\right\}$ of the set $N$ into $k$ subsets denotes a cooperation structure within the grand coalition $N$, i.e., players in set $T$ cooperate if and only if $T \in \mathcal{P}$. Then a PFF-game assigns a worth $v(S, \mathcal{P})$ to for every pair $(S, \mathcal{P})$ such that $S \in \mathcal{P}$, i.e., the worth of a coalition $S$ in $\mathcal{P}$ of $N$ depends on the cooperation structure $\mathcal{P} \backslash\{S\}$ of the players in $N \backslash S$. For $S \in \mathcal{P}$, the worth $v(S, \mathcal{P})$ denotes the amount that the players in $S$ can guarantee themselves by cooperating, when the players outside $S$ form coalitions $T, T \in \mathcal{P} \backslash\{S\}$. 


\section{River games}

\subsection{The basic river game}

In their paper 'Sharing a river', Ambec and Sprumont (2002) consider the problem to find a 'fair' distribution of the welfare resulting from allocating the water flows of an international river to the agents (e.g. firms, cities, countries) located along the river from upstream to downstream. Let $N=\{1, \ldots, n\}$ be the set of players representing the agents, in the sequel also called countries, along the river, numbered successively from upstream to downstream, and let $e_{i} \geq 0$ be the inflow of water on the territory of agent $i, i=1, \ldots, n$. Every agent is assumed to have a quasi-linear utility function given by $u^{i}\left(x_{i}, t_{i}\right)=b^{i}\left(x_{i}\right)+t_{i}$, where $t_{i}$ is a monetary compensation to agent $i, x_{i}$ is the amount of water allocated to agent $i$, and $b^{i}: \mathbb{R}_{+} \rightarrow \mathbb{R}$ is a continuous function yielding the benefit $b^{i}\left(x_{i}\right)$ to agent $i$ of the consumption $x_{i}$ of water. Ambec and Sprumont (2002) make the following assumption.

Assumption 3.1 In the river game every benefit function $b^{i}: \mathbb{R}_{+} \rightarrow \mathbb{R}$ is a strictly increasing and strictly concave function, which is differentiable for $x_{i}>0$ with derivative going to infinity as $x_{i}$ tends to zero.

Because of the one-directionality of the water from upstream to downstream, every agent can be assigned at most the water inflow at the territories of himself and his upstream agents, but the water inflow downstream of some agent can not be allocated to this agent. Therefore a water allocation $x \in \mathbb{R}_{+}^{n}$ assigns an amount of water $x_{i}$ to agent $i, i=1, \ldots, n$, under the restrictions

$$
\sum_{i=1}^{j} x_{i} \leq \sum_{i=1}^{j} e_{i}, \quad j=1, \ldots, n,
$$

i.e., for every agent $j$, the sum of the water assignments $x_{1}, \ldots, x_{j}$ is at most equal to the sum of the inflows $e_{1}, \ldots, e_{j}$. A water allocation $x$ yields total welfare $\sum_{i=1}^{n} b^{i}\left(x_{i}\right)$. A compensation scheme $t \in \mathbb{R}^{n}$ gives a monetary compensation $t_{i}$ to agent $i, i=1, \ldots, n$, under the restriction

$$
\sum_{i=1}^{n} t_{i} \leq 0 \text {. }
$$

So, the sum of all positive compensations (agents that receive money) is at most equal to the absolute value of the sum of all negative compensations (agents that have to pay). A welfare distribution is a pair $(x, t)$ of a water allocation $x$ and a compensation scheme $t$ yielding utility $u^{i}\left(x_{i}, t_{i}\right)=b^{i}\left(x_{i}\right)+t_{i}$ to every agent $i, i=1, \ldots, n$. A welfare distribution 
is Pareto efficient if no water and no money is wasted. So $(x, t)$ is Pareto efficient if and only if $x \in \mathbb{R}_{+}^{n}$ maximizes the welfare maximization problem

$$
\max _{x_{1}, \ldots, x_{n}} \sum_{i=1}^{n} b^{i}\left(x_{i}\right) \text { s.t. } \sum_{i=1}^{j} x_{i} \leq \sum_{i=1}^{j} e_{i}, \quad j=1, \ldots, n, \text { and } x_{i} \geq 0, i=1, \ldots, n,
$$

and the compensation scheme is budget balanced:

$$
\sum_{i=1}^{n} t_{i}=0
$$

Let $x^{*}$ be a solution of problem (3.1). Then a Pareto efficient welfare distribution $\left(x^{*}, t\right)$ yields payoffs (utilities)

$$
z_{i}=b^{i}\left(x^{*}\right)+t_{i}, \quad i=1, \ldots, n,
$$

with sum of payoffs equal to the Pareto efficient total welfare $\sum_{i=1}^{n} b^{i}\left(x_{i}^{*}\right)$.

The problem to find a 'fair' distribution of the Pareto efficient total welfare can be modeled by the following TU-game $(N, v)$. Obviously, the worth $v(N)$ is given by $v(N)=\sum_{i=1}^{n} b^{i}\left(x_{i}^{*}\right)$. Further, for any pair of agents $i, j$ with $j>i$, it holds that water inflow entering the river before the upstream agent $i$ can only be allocated to the downstream agent $j$ if every agent $h$ between agents $i$ and $j$ cooperates. Otherwise, since every benefit function $b^{h}$ is strictly increasing in $x_{h}$, every agent between $i$ and $j$ can increase its utility by taking the flow from $i$ to $j$ for its own use. Hence, a coalition $T$ is admissible if and only if $T$ is consecutive, i.e., $T=\{i, i+1, \ldots, j\}$ for some $i, j \in N, i \leq j$. In the sequel we denote such a coalition of consecutive agents by $[i, j]$. For any consecutive coalition $[i, j]$ its worth $v([i, j])$ is given by

$$
\begin{aligned}
& v([i, j])=\sum_{h=i}^{j} b^{h}\left(x_{h}^{[i, j]}\right) \quad \text { where } x^{[i, j]}=\left(x_{h}^{[i, j]}\right)_{h=i}^{j} \text { solves } \\
& \max _{x_{i}, \ldots, x_{j}} \sum_{h=i}^{j} b^{h}\left(x_{h}\right) \text { s.t. } \quad \sum_{k=i}^{l} x_{k} \leq \sum_{k=i}^{l} e_{k}, \quad l=i, \ldots, j, \text { and } x_{k} \geq 0, k=i, \ldots, j .
\end{aligned}
$$

So, the worth of a consecutive coalition is obtained by solving a similar maximization problem as for the 'grand coalition' $N$ in (3.1) but restricted to the water inflows and benefits of agents in the coalition. For any other (non-consecutive) coalition $S$, the worth $v(S)$ is equal to the sum of the worths of its maximal consecutive subsets. ${ }^{5}$ For benefit functions satisfying Assumption 3.1, we refer to this game introduced in Ambec and Sprumont (2002), as the basic river game and denote the collection of all basic river games on $N$

\footnotetext{
${ }^{5}$ A subset $T$ of $S$ is maximal consecutive if $T$ is consecutive and $T \cup h$ is not consecutive for any $h \in S \backslash T$.
} 
by $\mathcal{G}_{b}^{N}$. It has been shown by Ambec and Sprumont (2002) that every basic river game is convex.

We can now apply any solution concept from cooperative game theory to find a distribution of the Pareto efficient total welfare. Notice that a solution $f$ assigns payoff vector $z=f(v) \in \mathbb{R}^{n}$ to game $v \in \mathcal{G}_{b}^{N}$, which can be implemented by the welfare distribution $\left(x^{*}, t\right)$ with $t_{i}=z_{i}-b^{i}\left(x_{i}^{*}\right), i=1, \ldots, n$. The fairness of such a distribution depends on the properties of the solution. A minimal requirement is that the solution should be core-stable, i.e., for every $v \in \mathcal{G}_{b}^{N}$ the payoff vector $f(v) \in \operatorname{Core}(v)$. We will discuss this in more detail in Section 4.

\subsection{River systems with multiple springs}

In this subsection we extend the river game analysis to rivers that start at different springs. Thus, we consider a river system with several side-rivers which originate at different springs and that merge together to one river. Further, each spring is identified by an agent, i.e. we consider the most upstream agent along a side river as its spring. Every agent has precisely one downstream neighbor (except the final most downstream agent), but agents can have multiple upstream neighbors, namely in case an agent is located where two or more rivers merge together. We denote the number of springs by $s$ and denote $O=\{1, \ldots, s\}$ as the set of agents located at some spring, i.e., agent $j$ is located at spring $j, j=1, \ldots, s$. Let $n>s$ be the total number of agents. We index the (unique) most downstream agent by $n$. For agent $k$, let $U^{k}$ denote the set of upstream neighbors of $k$. Every agent $i \in N \backslash\{n\}$ is in exactly one $U^{k}$ for some $k \in N$. Notice that the structure of the river system is fully determined by the $n$-tuple of sets $\left(U^{k}\right)_{k \in N}$, with $U^{k}=\emptyset$ if and only if $k \in O$. We denote this $n$-tuple by $\mathcal{U}$. A pair $(N, \mathcal{U})$ consisting of a set of agents $N$ and a river structure $\mathcal{U}$ is then called a river system.

Example 3.2 Let $(N, \mathcal{U})$ represent a river system with $N=\{1,2,3,4,5,6,7\}$ and $U^{1}=$ $U^{2}=U^{3}=\emptyset, U^{4}=\{3\}, U^{5}=\{2,4\}, U^{6}=\{1,5\}$ and $U^{7}=\{6\}$, see Figure 1. So, $O=\{1,2,3\}$ is the set of springs and $n=7$ is the (unique) most downstream agent. The two rivers originating at 2 and 3 merge together at agent 5 and then this stream merges together at agent 6 with the side river originating at agent 1.

For $k \in N$, let $P^{k}$ denote the set of all agents upstream of $k$, including $k$ itself. Clearly, (i) $U^{k} \subseteq P^{k} \backslash\{k\}$ for every $k \in N$, (ii) $P^{k}=\{k\}$ and $U^{k}=\emptyset$ if $k \in O$, and (iii) $P^{n}=N$. Further, denote $N_{k}=N \backslash P^{k}$, i.e., $N_{k}$ is the complement of the set $P^{k}$ consisting of the set of agents not in $P^{k}$. Thus $N_{k}$ contains all agents downstream to agent $k$ and also all springs $j \in O \backslash P^{k}$ that are not upstream of $k$ and all agents downstream of 


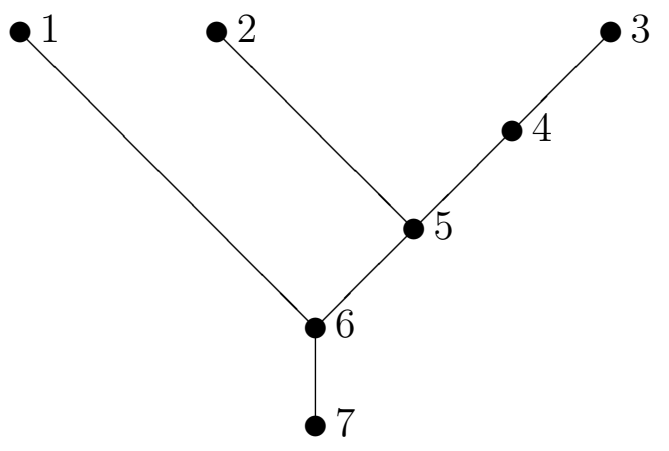

Figure 1: River structure from Example 3.2

these springs. Notice that for every agent $k$, both the $\left|P_{k}\right|-$ tuple $^{6}\left(U^{i} \cap P^{k}\right)_{i \in P^{k}}=\left(U^{i}\right)_{i \in P^{k}}$ and the $\left|N_{k}\right|$-tuple $\left(U^{i} \cap N_{k}\right)_{i \in N_{k}}$ are also river structures, so the pairs $\left(P_{k},\left(U^{i}\right)_{i \in P^{k}}\right)$ and $\left(N_{k},\left(U^{i} \cap N_{k}\right)_{i \in N_{k}}\right)$ are sub-river systems on the sets $P^{k}$, respectively $N_{k}$. Finally, let $Q_{k}$ denote the set of all agents downstream to agent $k$, including $k$ itself, and for $k \neq n$, let $d_{k}$ be the unique downstream neighbor of $k$. Taking $k=5$ in Example 3.2, $P^{5}=\{2,3,4,5\}$, $N_{5}=\{1,6,7\}, Q_{5}=\{5,6,7\}$ and $d_{5}=6$. Further, the sub-river system $\left(N_{5},\left(U^{i} \cap N_{5}\right)_{i \in N_{5}}\right)$ is given by the river structure $U^{1} \cap N_{5}=\emptyset, U^{6} \cap N_{5}=\{1\}$ and $U^{7} \cap N_{5}=\{6\}$.

We assume again that each agent has a quasi-linear utility function given by $u^{i}\left(x_{i}, t_{i}\right)$ $=b^{i}\left(x_{i}\right)+t_{i}$ and that Assumption 3.1 holds. Now we slightly have to adapt the constraint in the definition of water allocation $x$ to

$$
\sum_{i \in P^{j}} x_{i} \leq \sum_{i \in P^{j}} e_{i}, \quad j=1, \ldots, n
$$

i.e., for every agent $j$, the sum of the water assignment uses of agent $j$ and all its upstream agents is at most equal to the sum of the inflows at $j$ and all its upstream agents, and correspondingly we have to modify the constraints in the welfare maximization problem (3.1).

Modeling the problem to find a 'fair' distribution of the Pareto efficient total welfare by a TU-game $(N, v)$ in case of multiple springs, we assume that a coalition of agents $S$ can cooperate together when (i) there exists a $k \in S$ such that $S \subseteq P^{k}$, i.e., there is a unique most downstream agent, and (ii) for every $i \in S \backslash\{k\}$, every agent between $i$ and $k$ is also in $S$. Condition (i) means that agents on two branches can not cooperate if they do not have a common most downstream agent, for instance in Example 3.2 the two upstream branches $\{1\}$ and $\{3,4\}$ can not benefit from cooperation in the coalition $\{1,3,4\}$. The second condition generalizes the notion of a coalition of consecutive agents to the case of multiple springs: it implies that when $j$ cooperates with an upstream agent $i$, every agent on the branch between $i$ and $j$ also cooperates. In Example 3.2, agents 2 and 6 can only

\footnotetext{
${ }^{6}$ For a set $A,|A|$ denotes the number of elements in $A$.
} 
cooperate when also 5 agrees. We say that a coalition $S$ is connected when $S$ satisfies (i) and (ii). Modeling this situation as a TU-game, the worth $v(S)$ of a connected coalition $S$ is given by

$$
\begin{aligned}
& v(S)=\sum_{h \in S} b^{h}\left(x_{h}^{S}\right) \text { where } x^{S}=\left(x_{h}^{S}\right)_{h \in S} \text { solves } \\
& \max _{\left\{x_{h} \geq 0 \mid h \in S\right\}} \sum_{h \in S} b^{h}\left(x_{h}\right) \text { s.t. } \sum_{i \in P^{j} \cap S} x_{i} \leq \sum_{i \in P^{j} \cap S} e_{i}, j \in S, \text { and } x_{i} \geq 0, i \in S .
\end{aligned}
$$

For any other (non-connected) coalition $S$, the worth $v(S)$ is equal to the sum of the worths of its maximally connected subsets. ${ }^{7}$

In particular we consider an agent $k$ which has at least two upstream neighbors, i.e., $k$ is an agent at which at least two rivers merge together. By definition the set $P^{k}$ is connected for every $k$, but at an agent $k$ with at least two upstream neighbors the set $P^{k} \backslash\{k\}$ is not connected (in Example 3.2 the set $P^{5} \backslash\{5\}=\{2,3,4\}$ is not connected, but it contains two maximal connected subsets: $\{2\}$ and $\{3,4\})$. For every $k \in N$ it holds that

$$
v\left(P^{k} \backslash\{k\}\right)=\sum_{j \in U^{k}} v\left(P^{j}\right) .
$$

The triple $(N, \mathcal{U}, v)$ completely describes a river game on a river system with multiple springs. When we take the river system $(N, \mathcal{U})$ as given, we denote the collection of all characteristic functions $v$ obtained from river situations on $(N, \mathcal{U})$ with benefit functions satisfying Assumption 3.1 by $\mathcal{G}^{(N, \mathcal{U})}$.

\subsection{River games with externalities}

Ambec and Ehlers (2008) have generalized the basic one-spring river game of Ambec and Sprumont by allowing for satiable agents. This means that Assumption 3.1 is weakened by deleting the requirement that the benefit function is strictly increasing.

Assumption 3.3 In the river game every benefit function $b^{i}: \mathbb{R}_{+} \rightarrow \mathbb{R}$ is a strictly concave function, which is differentiable for $x_{i}>0$ with derivative going to infinity as $x_{i}$ tends to zero.

Hence, either $b^{i}$ is strictly increasing, or there exists a unique number, say $s^{i}$, such that $b^{i}$ is strictly increasing on $x_{i}<s^{i}$ and strictly decreasing when $x_{i}>s^{i}$, thus the derivative of $b^{i}$ is zero at point $s^{i}$. The point $s^{i}$ is the satiation point of agent $i$. We now consider a river

\footnotetext{
${ }^{7}$ A subset $T$ of $S$ is maximal connected in $S$ if $T$ is connected in the sub-river system $\left(S,\left(U^{k} \cap S\right)_{k \in S}\right)$ and $T \cup h$ is not connected in this sub-river system for any $h \in S \backslash T$.
} 
with multiple springs and each agent having a benefit function satisfying Assumption 3.3. Without loss of generality (see Ambec and Ehlers, 2008), we further assume that $e_{i}<s^{i}$ for all $i$ (with $s^{i}=\infty$ when $b^{i}$ is strictly increasing). ${ }^{8}$

The existence of satiation points has serious consequences for the resulting game. Before, under Assumption 3.1, only connected coalitions were able to cooperate because any water transfered from one part of a non-connected coalition to another would fully be consumed by 'intermediary' agents. So, a non-connected coalition $S$ consisting of two connected subsets of agents, say an upstream connected subset $S_{1}$ and a downstream connected subset $S_{2}$, would never transfer water from $S_{1}$ to $S_{2}$ because the strictly increasing benefit functions of the agents would make that all water sent from $S_{1}$ to $S_{2}$ would immediately be taken by the agents in-between $S_{1}$ and $S_{2}$. In contrast, under Assumption 3.3 it might be profitable for a non-connected coalition to transfer water between its nonconnected subsets. When all agents in-between $S_{1}$ and $S_{2}$ have a satiation point then it might be profitable for the not connected coalition $S=S_{1} \cup S_{2}$ to send water from its upstream part $S_{1}$ to its downstream part $S_{2}$. Although some of this flow might be taken by the in-between agents, these agents will only take water up to their satiation points. So, when the flow is big enough, part of it will reach $S_{2}$, possibly rendering cooperation between the two non-connected parts of the coalition profitable. As a result of the change in the assumption, the worth of an non-connected coalition $S$ can now be higher than the sum of the worths of its maximal connected subsets.

This phenomenon might cause positive externalities on a connected coalition $T$. Under Assumption 3.1, the worth of $T$ follows from the maximization program (3.3). However, when all agents in $T$ have satiation points, it might be profitable for agents upstream of $T$ to send water to agents downstream of $T$. Thus the worth of $T$ depends on the coalition formation of the agents outside $T$. The problem to find a fair distribution of the Pareto efficient total welfare can now be modeled by a cooperative game in partition function form. As mentioned in the preliminaries, such a game assigns a worth $v(S, \mathcal{P})$ to every coalition $S$ in a coalition structure $\mathcal{P}$ on $N$. This worth denotes the maximum welfare (sum of the benefits) that the agents in coalition $S$ can guarantee themselves by cooperating, when the agents outside $S$ form coalitions $T, T \in \mathcal{P} \backslash\{S\} .{ }^{9}$ When we take the river system $(N, \mathcal{U})$ as given, we denote the collection of all partition function form games $v$ on $(N, \mathcal{U})$ with benefit functions satisfying Assumption 3.3 by $\mathcal{P} \mathcal{G}^{(N, \mathcal{U})}$.

When $S \in \mathcal{P}$ and every coalition $T \in \mathcal{P}, T \neq S$, is a singleton, then the agents outside $S$ act individually and every $i \notin S$ consumes at least its own water inflow $e_{i}$

\footnotetext{
${ }^{8}$ If $e_{i}>s_{i}$ then in the optimal allocation problem $e_{i}-s_{i}$ can be considered as additional inflow at its downstream neighbor $d_{i}$.

${ }^{9}$ For a river with one spring, Ambec and Ehlers (2008) provides an iterative procedure to find the worths $v(S, \mathcal{P})$ for every $\mathcal{P}$ and every $S \in \mathcal{P}$.
} 
(because $e_{i}<s^{i}$ for all $i$ ). We denote $\mathcal{P}_{S}=\{S\} \cup\{\{i\}, i \notin S\}$ as the partition where all agents outside $S$ do not cooperate and act as singletons, and $v_{*}(S)=v\left(S, \mathcal{P}_{S}\right)$ as the corresponding worth in the partition function form game. This worth is called (Ambec and Ehlers, 2008) the (non-cooperative) core lower bound of $S$. For a connected coalition we have that $v_{*}(S)=v(S)$, i.e., $v_{*}(S)$ is precisely the worth that $S$ can obtain by solving the welfare maximizing problem (3.3) and thus is equal to the welfare that the agents in $S$ obtain when allocating optimally their own water inflows amongst themselves. We further denote $v^{*}(S)=v\left(S, \mathcal{P}^{S}\right)$, with $\mathcal{P}^{S}=\{S, N \backslash S\}$ the partition such that all agents not in $\mathrm{S}$ work together. The worth $v^{*}(S)$ is called the (cooperative) core upper bound of $S$. It is the amount that the agents in $S$ can guarantee themselves when the agents outside $S$ cooperate together in coalition $N \backslash S$. Notice that $v_{*}(S)$ and $v^{*}(S)$ are defined for every $S$, not only for connected coalitions. Also notice that $v_{*}(N)=v^{*}(N)=v(N,\{N\})=v(N)$ is the worth of the grand coalition when all agents cooperate together.

The following results have been stated in Ambec and Ehlers (2008) for rivers with one spring and generalize straightforwardly to rivers with multiple springs.

Lemma 3.4 Let $\mathcal{P}$ be a partition of $N$ and $S \in \mathcal{P}$. Then

(i) $v_{*}(S) \leq v(S, \mathcal{P})$

(ii) For any two different $S, T \in \mathcal{P}, v(S, \mathcal{P})+v(T, \mathcal{P}) \leq v\left(S \cup T, \mathcal{P}^{\prime}\right)$ with $\mathcal{P}^{\prime}=(\mathcal{P} \backslash$ $\{S, T\}) \cup\{S \cup T\}$.

Notice that (i) also implies that $v_{*}(S) \leq v^{*}(S)$ and that (ii) implies that

$$
v_{*}(S)+v_{*}(T) \leq v_{*}(S \cup T)
$$

for every disjoint $S$ and $T$. Hence, the worths $v_{*}(S), S \subseteq N$, induce a superadditive TUgame $\left(N, v_{*}\right)$. Recall that in the case without externalities the above inequality holds with equality when $S$ and $T$ are two disjoint connected coalitions and $S \cup T$ is not connected. This does not need to be true in the river game with satiation points. For instance when $S$ is upstream of $T$, the union $S \cup T$ may benefit from a water flow going from $S$ to $T$. For rivers with one spring it is also stated in Ambec and Ehlers (2008) that for coalitions $S=[1, i], i=1, \ldots, n$,

$$
v([1, i], \mathcal{P})=v_{*}([1, i]) \text { for every } \mathcal{P} \text { with }[1, i] \in \mathcal{P},
$$

i.e., when coalition $S$ consists of some agent $i$ and all its upstream agents, then the worth of $S$ does not depend on the partition of the agents outside $S$. Indeed, by definition, the worth of such an upstream coalition $S$ does not depend on the behavior of the agents downstream of $S$. In case of a river with multiple springs, this result generalizes to every upstream coalition $P^{k}$ and its complement $N_{k}=N \backslash P^{k}, k \in N$. 
Theorem 3.5 Let $v \in \mathcal{P} \mathcal{G}^{(N, \mathcal{U})}$ be a partition function form river game on $(N, \mathcal{U})$. Then, for every $\mathcal{P}$ and $S \in \mathcal{P}$,

$$
v(S, \mathcal{P})=v_{*}(S) \text { if } S=P^{k} \text { or } S=N_{k}, \text { for some } k \in N \text {. }
$$

Proof. Let $S=P^{k}$ for some $k \in N$. Since $P^{k}$ consists of agent $k$ and all its upstream agents, its worth $v\left(P^{k}, \mathcal{P}\right)$ does not depend on the partition $\mathcal{P} \backslash\left\{P^{k}\right\}$ of the agents outside $P^{k}$. Hence $v\left(P^{k}, \mathcal{P}\right)=v_{*}\left(P^{k}\right)$ for all $\mathcal{P}$ with $P^{k} \in \mathcal{P}$.

Next consider $S=N_{k}$ for some $k \in N$. By definition of $P^{k}$, agent $k$ is the only agent in $P^{k}$ that is connected to an agent in $N_{k}$, namely to its unique downstream neighbor $d_{k}$. Further, by definition of $P^{k}$, there are no agents in $P^{k}$ downstream of $k$. By the assumption that $e_{h}<s^{h}$ for every $h \in N$, it follows that agent $d_{k}$ never receives any water from $k$, independent of the partition of $P^{k}=N \backslash N_{k}$. So, $v\left(N_{k}, \mathcal{P}\right)=v_{*}\left(N_{k}\right)$ for all $\mathcal{P}$ with $N_{k} \in \mathcal{P}$.

We say that the worth of every coalition of type $P^{k}$ or type $N_{k}$ is externality-free, i.e., the worth does not depend on the partition of the agents outside a coalition of such a type.

\section{Welfare distribution in international river basins}

In this section we restrict the analysis for river systems with agents satisfying Assumption 3.1 and discuss the implications of principles for water allocation for the properties to be satisfied by solutions on the class $\mathcal{G}^{(N, \mathcal{U})}$ of river games with (possibly) multiple springs but without externalities. We return to the case of externalities in Section 5.

\subsection{Efficiency and the TIBS principle}

In Kilgour and Dinar (1995) several principles have been proposed to prevent or resolve disputes on water allocation within an international water basin. Since there is no binding international law governing the allocation of water in international rivers, these principles, in combination with a legal text called the 1966 Helsinki Document (see Kilgour and Dinar), are the only guidelines that are available in determining a 'fair' welfare distribution and thus a 'fair' solution for the class of cooperative TU-games on river systems with multiple springs. ${ }^{10}$

According to the 1966 Helsinki Document two considerations that are to be included in resolving water disputes are 'efficiency of the water use' and 'Pareto optimality'. In the

\footnotetext{
${ }^{10}$ These principles can, of course, also be used when the agents in the model are not countries but, for instance, firms or cities.
} 
context of this paper we can translate these considerations into an axiom that we can then use (in combination with other axioms) to find a solution for a river game. Efficiency of water use and Pareto optimality imply that the grand coalition optimally allocates its own water inflow amongst its own members and that the monetary compensations are budget balanced. So, translating efficiency of water use and Pareto optimality into an axiom for a solution $f$ gives us straightforwardly the following axiom to be satisfied by a solution $f$ on $\mathcal{G}^{(N, \mathcal{U})}$.

\section{Axiom 4.1 Efficiency}

$A$ solution $f$ on the class of river games $\mathcal{G}^{(N, \mathcal{U})}$ is efficient if $\sum_{i \in N} f_{i}(v)=v(N)$ for every $v \in \mathcal{G}^{(N, \mathcal{U})}$.

This axiom implies for any river game on $(N, \mathcal{U})$ that the agents efficiently distribute the worth of the grand coalition $v(N)$ (the maximum utility they can obtain when they all cooperate).

The principles discussed in Kilgour and Dinar (1995) concern the 'water rights' assigned to the different countries along the river. The principle of Absolute Territorial Sovereignty (ATS), or Harmon doctrine, states that a country has absolute sovereignty over the area of any river basin on its territory. This principle clearly favors upstream countries in that it allows countries to use any water that flows into the river on their territory without taking into account what consequences this might have for its downstream countries. For a solution $f$ on the class $\mathcal{G}^{(N, \mathcal{U})}$ of river games this principle implies that for every connected coalition of agents, the agents can secure themselves at least the welfare level that can be reached by allocating optimally the water that they control amongst themselves (given the one-directionality of the flow). So, for every connected coalition $S$ it should hold that $\sum_{i \in S} f_{i}(v) \geq v(S)$. Then this condition also holds for every not connected subset, because for such a set the worth $v(S)$ is equal to the sum of the worths of its maximally connected subsets. So, the ATS property results in the requirement that the welfare distribution should be core-stable.

The second doctrine listed in Kilgour and Dinar (1995, p.1) is:

"The principle of Territorial Integration of all Basin States. Symmetrically, this principle favors downstream states, to which it accords "equal" use, without regard to their contribution to the flow".

This TIBS-principle does not make any country the legal owner of water. Instead, it states that the river water belongs to all the countries combined, no matter where it enters the river, and that each country has the right to 'a share' of the total amount of water. Of course, also with respect to this principle we have to take into account the 
one-directionality of the water flow. For instance we can not give agent 1 (at spring one by the convention on the indexing of the agents in this paper) a right to more water than actually enters the river at its own territory. However notice that, while agent 1 can never obtain more water than enters the river on his own territory, he can reach a higher utility level than the utility it obtains from consuming his own inflow because he has the option to 'trade' some of the water with its downstream neighbor (that possibly derives a higher utility from the water) in exchange for a monetary compensation. While ATS has a clear implication for solutions, namely core stability, TIBS can be interpreted in several ways. Since water cannot be allocated upstream, we interpret the TIBS principle as follows: given some agent $j \in N$, this agent and all his downstream agents are entitled to receive a share of the water inflow at the territory of agent $j$. It is not difficult to see that the TIBS principle favors downstream countries more than the ATS principle.

An extreme interpretation of the TIBS principle, called Unlimited Territorial Integrity (UTI), states that country $j$ can claim at most a utility equal to the benefit of the total inflow of water at the territories of country $j$ itself and all its upstream countries. For the basic river game with a single spring Ambec and Sprumont (2002) conclude from the UTI principle that the welfare distribution should satisfy the property that no coalition $S$ should get a total welfare above its aspiration level, defined as the highest sum of all benefits over the agents in $S$ that can be obtained by an optimal allocation among its own members of all the water inflows at all agents along the river from 1 to $s$, where $s$ is the most downstream agent of coalition $S$. So, the aspiration level is the welfare level that can be obtained when the agents in $S$ can also use the water inflows of the agents not in $S$, but upstream to the most downstream member of $S$. Applying this to the upstream coalition of consecutive agents from 1 to $j$, this aspiration level fairness property requires that the solution of the cooperative TU-game gives a total payoff to coalition $[1, j]$ at most equal to the aspiration level upperbound $v([1, j])$. On the other hand, core-stableness requires that coalition $[1, j]$ receives at least $v([1, j])$. Therefore, core-stableness and fairness imply that the total payoff to the agents in coalition $[1, j]$ should be equal to $v([1, j])$. From this Ambec and Sprumont (2002) conclude that there is a unique welfare distribution that satisfies both the core lower bounds and the aspiration level upper bounds, being the downstream incremental welfare distribution that is given by the marginal vector of the game $v$ (see Section 2) with respect to the permutation $\pi$ on $N$ given by $\pi(i)=i, i \in N$, i.e. where the agents enter from upstream to downstream. In the sequel we denote the solution that assigns this marginal vector to any basic river game in the class $\mathcal{G}_{b}^{N}$ by $f^{d}$. So the solution $f^{d}$ assigning the marginal contributions

$$
f_{1}^{d}(v)=v(1) \text { and } f_{i}^{d}(v)=v([1, i])-v([1, i-1]), \quad i=2, \ldots, n,
$$

yields for every $v \in \mathcal{G}_{b}^{N}$ the downstream incremental welfare distribution. Notice that it 
indeed follows from the convexity of every game $v$ in this class that $f^{d}(v)$ is core-stable.

The downstream incremental welfare distribution has the property that for every $i<$ $n$, the total payoff of the agents in the consecutive coalition $[1, i]$ upstream of $i$ (including $i$ itself) is equal to $v([1, i])$, while the total payoff to the downstream coalition $[i+1, n]$ is equal to $v(N)-v([1, i]) \geq v([i+1, n])$. In fact, all additional welfare that is realised when the two coalitions $[1, i]$ and $[i+1, n]$ merge to the grand coalition $N$ goes to the downstream coalition. However, any upstream coalition $[1, i]$ can prevent that coalition $[i+1, n]$ gets more than $v([i+1, n])$ by using all its inflows $e_{1}, \ldots, e_{i}$ by itself. This is the opposite extreme interpretation of the TIBS principle saying that country $i$ is entitled to receive at least all welfare gains from allocating optimally his own water inflow among himself and all his downstream agents. In Herings, van der Laan and Talman (2007) and van den Brink, van der Laan and Vasil'ev (2007) it is alternatively argued that a coalition $[1, i]$ can play some type of ultimatum game by claiming that they will use their total water inflow $\sum_{h=1}^{i} e_{i}$ by themselves, unless the agents of the downstream coalition $[i+1, j]$ agree to make a monetary compensation almost equal to the total welfare gain of cooperation. This results in precisely the opposite of the solution proposed by Ambec and Sprumont, namely the upstream incremental welfare distribution, being the welfare distribution given by the marginal vector with respect to the permutation $\pi$ on $N$ given by $\pi(i)=n-i+1$, $i \in N$, i.e. where the agents enter from downstream to upstream. In the sequel we denote the solution that assigns this marginal vector to any basic river game in the class $\mathcal{G}_{b}^{N}$ by $f^{u}$, being the solution assigning the marginal contributions

$$
f_{n}^{u}(v)=v(n) \text { and } f_{i}^{u}(v)=v([i, n])-v([i+1, n]), \quad i=2, \ldots, n .
$$

Although this solution does not satisfy the aspiration level upper bounds, it is also corestable. For these reasons Herings, van der Laan and Talman (2007) and van den Brink, van der Laan and Vasil'ev (2007) assert that the solution $f^{u}$ is at least as reasonable as its counterpart $f^{d}$.

Whereas the above two interpretations of the TIBS principle are rather extreme, in this paper we look at solutions that can be motivated by the TIBS principle in a broader sense, including the above two extreme interpretations. To do so we have to translate the TIBS principle into an axiom just as we did for the two considerations from the 1966 Helsinki Document. The general TIBS principle states that given some agent $j \in N$, this agent and all its downstream agents are entitled to receive 'a share' of the water inflow at agent $j$. In this paper water and welfare are interchangeable (water provides welfare through the benefit function). To say that a certain country has the right to a certain amount of water comes down to saying that a country has the right to a certain share in the welfare. The TIBS principle therefore implies that each agent has the right to a specific 
share in welfare (depending on the share of water that it has the right to according to the principle).

To translate this principle in a fairness axiom for a solution on the class of TU-games $\mathcal{G}^{(N, \mathcal{U})}$, suppose that the agents along the river are cooperating according to the coalition structure $\mathcal{P}=\left\{P^{i}, N_{i}\right\}$ for some agent $i \in N \backslash\{n\}$. So, agent $i$ is cooperating with all its upstream agents in coalition $P^{i}$, while all other agents are cooperating in its complement coalition $N_{i}$. This can happen, for instance, because agent $i$, for some reason or another, is not willing to send water to its unique downstream neighbor $d_{i}$. In this situation the agents in $P^{i}$ distribute their total welfare $v\left(P^{i}\right)$ and the agents in $N_{i}$ distribute $v\left(N_{i}\right)$. The question that can then be asked is: how should the gain in welfare $v(N)-v\left(P^{i}\right)-v\left(N_{i}\right)$ that is created by joining the two coalitions be divided among the agents? Evidently, we can ask this question for each agent $i \in N \backslash\{n\}$. The TIBS principle provides us with an answer to this question. Let $\alpha \in \mathbb{R}_{+}^{n}$ with $\sum_{h \in N} \alpha_{h}=1$ be a vector of weights, with $\alpha_{h} \geq 0$ the weight of agent $h, h \in N$. Then we interpret the TIBS principle by saying that the gain in welfare that is created by joining the two coalitions $P^{i}$ and $N_{i}$, should be divided among these two coalitions proportional to the sum of the weights in these two coalitions. Denoting $\alpha_{S}=\sum_{i \in S} \alpha_{i}$ for every $S \subseteq N$, we thus require that

$$
\frac{\sum_{k \in P^{i}} f_{k}(v)-v\left(P^{i}\right)}{\sum_{k \in N_{i}} f_{k}(v)-v\left(N_{i}\right)}=\frac{\alpha_{P^{i}}}{\alpha_{N_{i}}}
$$

assuming that both $\alpha_{P^{i}}$ and $\alpha_{N_{i}}$ are nonzero. This leads to the following fairness axiom (which also is valid in case some weights are zero) for efficient solutions on the class of river games.

\section{Axiom 4.2 $\alpha$-TIBS Fairness}

Let $\alpha \in \mathbb{R}_{+}^{n}$ be such that $\sum_{i \in N} \alpha_{i}=1$. An efficient solution $f$ on the class of river games $\mathcal{G}^{(N, \mathcal{U})}$ satisfies $\alpha$-TIBS fairness if, for every $v \in \mathcal{G}^{(N, \mathcal{U})}$ and any $i \in N \backslash\{n\}$, it holds that

$$
\alpha_{N_{i}}\left(\sum_{k \in P^{i}} f_{k}(v)-v\left(P^{i}\right)\right)=\alpha_{P^{i}}\left(\sum_{k \in N_{i}} f_{k}(v)-v\left(N_{i}\right)\right) .
$$

Notice that the TIBS principle speaks about agents having the right to 'a share' of the water inflow, but does not specify the shares and even does not require the shares to be equal. Therefore we allow for any nonnegative weight vector $\alpha$ which components add up to one. For the specific case that all weights are equal, i.e., $\alpha_{i}=\frac{1}{n}$ for all $i \in N, \alpha$-TIBS fairness yields the following equal weights fairness axiom. ${ }^{11}$

\footnotetext{
${ }^{11}$ This axiom is similar to the so-called component fairness axiom of Herings, van der Laan and Talman (2008) within the more general context of games on cycle-free graph structures (trees). In Béal, Rémila and Solal (2009) this component fairness axiom is generalised to weighted component fairness for forest games. However, this generalization differs from the $\alpha$-TIBS fairness, because it assigns weights to so-called cones (of a tree) instead of assigning weights to individual players.
} 


\section{Axiom 4.3 Equal Weights TIBS Fairness}

An efficient solution $f$ on the class of river games $\mathcal{G}^{(N, \mathcal{U})}$ satisfies equal weights TIBS fairness if, for every $v \in \mathcal{G}^{(N, \mathcal{U})}$ and any $i \in N \backslash\{n\}$, it holds that

$$
\frac{1}{\left|P^{i}\right|}\left(\sum_{k \in P^{i}} f_{k}(v)-v\left(P^{i}\right)\right)=\frac{1}{\left|N_{i}\right|}\left(\sum_{k \in N_{i}} f_{k}(v)-v\left(N_{i}\right)\right) .
$$

\subsection{Weighted hierarchical solutions}

The introduction of efficiency and $\alpha$-TIBS fairness allows us to find a class of 'fair' (according to these principles) solutions for river games without externalities in the class $\mathcal{G}^{(N, \mathcal{U})}$. We call this the class of weighted hierarchical solutions. Each solution from this class assigns to every river game a weighted hierarchical outcome. The notion of hierarchical outcome has been introduced by Demange (2004) in the context of games on cycle-free graph structures. In this same context Herings, van der Laan and Talman (2008) propose the average of all hierarchical outcomes as the so-called average tree solution, whereas Béal, Rémila and Solal (2010) generalize this solution to the class of all weighted averages of the hierarchical outcomes. In this section we examine the class of weighted hierarchical solutions from the perspective of the river game without referring to the underlying graph-theoretical concepts.

For an agent $i \in N$ and an agent $k$ downstream of $i$, let $k^{i}$ be the last agent before $k$ on the river branch from $i$ to $k$ (with $k^{i}=i$ when $k=d_{i}$ ). Now take some agent $i \in N$ and for every $k \in N$, consider the following payoff $t_{k}^{i}(v)$ for agent $k$ (recall from Section 3 that $Q_{i}$ is the set of agents downstream of $i$, including $i$ itself) given by

$$
t_{k}^{i}(v)= \begin{cases}v\left(P^{k}\right)-v\left(P^{k} \backslash\{k\}\right) & \text { if } k \in N \backslash Q_{i}, \\ v\left(N_{k^{i}}\right)-v\left(N_{k^{i}} \backslash\{k\}\right) & \text { if } k \in Q_{i} \backslash\{i\}, \\ v(N)-v\left(P^{k} \backslash\{k\}\right)-v\left(N_{k}\right) & \text { if } k=i .\end{cases}
$$

The payoff vector $t^{i}(v)$ gives a hierarchical outcome of Demange (2004) when $i$ is taken to be 'top' agent in the hierarchy. The set of agents $N \backslash Q_{i}$ consists of all agents upstream to $i$ and all agents neither upstream nor downstream of $i$. For instance, in Example 3.2 the set $N \backslash Q_{5}$ consists of the agents 2, 3 and 4 upstream of 5 and agent 1 , which is neither upstream nor downstream of 5 . Each agent $k$ not in $Q_{i}$ receives his marginal contribution to the coalition of agents $P^{k}$ consisting of this agent $k$ and all his upstream agents. For an agent $k$ downstream of agent $i$ we consider his upstream neighbor $k^{i}$ on the branch to $i$. Then such an agent $k$ receives his marginal contribution to the set $N_{k^{i}}$, that is the marginal contribution to the set of agents who can be reached from $k$ by walking along the river without visiting his upstream neighbor $k^{i}$ on the branch from $k$ to $i$. Notice that $k=d_{k^{i}}$, i.e., $k$ himself is the (unique) downstream neighbor of $k^{i}$. Finally, 
top agent $i$ receives $v(N)-v\left(P^{i} \backslash\{i\}\right)-v\left(N_{i}\right)$. Notice that the sets $P^{i} \backslash\{i\}$ of all agents upstream of $i$ and the set $N_{i}$ of all agents not in $P^{i}$ can not cooperate without $i$ and thus $v(N \backslash\{i\})=v\left(P^{i} \backslash\{i\}\right)+v\left(N_{i}\right)$. So, $t_{i}^{i}(v)=v(N)-v(N \backslash\{i\})$, i.e., top agent $i$ receives his marginal contribution to the grand coalition $N$, which is equal to the additional welfare that he generates by joining together the two coalitions $P^{i} \backslash\{i\}$ and $N_{i}$.

We can consider agent $i$ as the top agent in a hierarchy on the set of all agents as follows. For an agent $h \neq i$, let $i_{h}$ be the distance from $i$ to $h$ in the river system $(N, \mathcal{U})$, with the distance defined as the number of agents (including $h$ itself, but not $i$ ) that has to be visited when going from $i$ to $h$ along the river. For example, taking $i=4$ in the river system of Figure 1, the distance to agent 3 is one, the distance to agent 2 is two and the distance to agents 1 or 7 is equal to three. Now, let $\pi^{i}$ be a permutation such that an agent $h \neq i$ enters before an agent $k \neq i$ if $i_{h}>i_{k}$ (and with agent $i$ as the last agent that enters). So, with $i=4$ in Figure 1 first agents 1 and 7 with distance three enter (in arbitrary order), then agents 2 and 6 with distance two, then agents 3 and 5 with distance one and finally agent 4 . Then it follows from the fact that for every $S$ the worth $v(S)$ is equal to the sum of the worths of its maximally connected subsets that $t^{i}(v)$ is the marginal vector of the game $v$ with respect to this order in which agent $i$ enters last. So, considering the river system as a 'hierarchy' with agent $i$ as the top agent, the agents enter successively in order of their distance to the top and receive their marginal contribution to the coalition of agents that entered before. Since every hierarchical outcome is equal to the marginal vector corresponding to such a permutation $\pi^{i}$, it follows by definition that every hierarchical outcome is efficient and thus the sum of the payoffs is equal to $v(N)$. This also follows straightforwardly by the fact that the top agent is the last agent that enters and receives everything that has not yet been assigned to the other agents.

Each agent $i \in N$ induces a hierarchical outcome $t^{i}(v)$, so that the total number of hierarchical outcomes is equal to $n$. For every nonnegative vector $\alpha \in \mathbb{R}_{+}^{n}$, with $\sum_{i \in N} \alpha_{i}=$ 1 , the payoff vector $h^{\alpha}(v) \in \mathbb{R}^{n}$ given by

$$
h^{\alpha}(v)=\sum_{i \in N} \alpha_{i} t^{i}(v)
$$

is a weighted hierarchical outcome of $v \in \mathcal{G}^{(N, \mathcal{U})}$. This gives us the next definition of a weighted hierarchical solution on $\mathcal{G}^{(N, \mathcal{U})}$.

Definition 4.4 A solution $f$ on the class of river games $\mathcal{G}^{(N, \mathcal{U})}$ is a weighted hierarchical solution if there exists $\alpha \in \mathbb{R}_{+}^{n}$ with $\sum_{i \in N} \alpha_{i}=1$, such that for every $v \in \mathcal{G}_{m}^{(N, \mathcal{U})}$

$$
f_{i}(v)=h_{i}^{\alpha}(v), \forall i \in N .
$$

We now prove that every weighted hierarchical solution is the unique efficient solution that satisfies the corresponding $\alpha$-TIBS fairness axiom. Therefore we first show some lemmas. 
Lemma 4.5 Given a top agent $j$, consider an agent $i$ and the hierarchical outcome $t^{j}(v)$. Then

(i) $\sum_{k \in P^{i}} t_{k}^{j}(v)=v(N)-v\left(N_{i}\right)$ if $j \in P^{i}$, and

(ii) $\sum_{k \in P^{i}} t_{k}^{j}(v)=v\left(P^{i}\right)$ if $j \in N_{i}$.

\section{Proof.}

(i) If $j \in P^{i}$, then for every $h \in N_{i}$, agent $i$ has to be passed when one goes along the river from $j$ to $h$. So, for every $h \in N_{i}$ the distance from $j$ to $h$ is bigger than the distance from $j$ to $i$, and thus every agent $h \in N_{i}$ enters before $i$ in every permutation $\pi^{j}$ that results in the marginal vector $t^{j}(v)$. Hence all agents of the set $N_{i}$ enter before $i$ and the total payoff $\sum_{h \in N_{i}} t_{h}^{j}(v)$ to these is equal to $v\left(N_{i}\right)$. From the efficiency it then follows that the total payment to the agents in the complementary set $P^{i}$ is equal to $v(N)-v\left(N_{i}\right)$.

(ii) If $j \in N_{i}$ then $k \in N \backslash Q_{j}$ for every $k \in P^{i}$. So every $k \in P^{i}$ receives payoff $t_{k}^{j}(v)=v\left(P^{k}\right)-v\left(P^{k} \backslash\{k\}\right)$. Summing up over all $k \in P^{i}$ yields that $\sum_{k \in P^{i}} t_{k}^{j}(v)=v\left(P^{i}\right)$.

Lemma 4.6 Let $\alpha \in \mathbb{R}_{+}^{n}$ be such that $\sum_{i \in N} \alpha_{i}=1$. Then the solution $h^{\alpha}$ on the class of river games $\mathcal{G}^{(N, \mathcal{U})}$ satisfies $\alpha$-TIBS fairness.

Proof. We distinguish three cases.

Case 1. Consider an agent $i \in N$ such that $\alpha_{P^{i}}>0$ and $\alpha_{N_{i}}>0$. Then

$$
\begin{aligned}
\sum_{k \in P^{i}} h_{k}^{\alpha}(v)-v\left(P^{i}\right) & =\sum_{k \in P^{i}} \sum_{j \in N} \alpha_{j} t_{k}^{j}(v)-v\left(P^{i}\right) \\
& =\sum_{k \in P^{i}} \sum_{j \in P^{i}} \alpha_{j} t_{k}^{j}(v)+\sum_{k \in P^{i}} \sum_{j \in N_{i}} \alpha_{j} t_{k}^{j}(v)-v\left(P^{i}\right) \\
& =\sum_{j \in P^{i}} \sum_{k \in P^{i}} \alpha_{j} t_{k}^{j}(v)+\sum_{j \in N_{i}} \sum_{k \in P^{i}} \alpha_{j} t_{k}^{j}(v)-v\left(P^{i}\right) \\
& =\sum_{j \in P^{i}} \alpha_{j} \sum_{k \in P^{i}} t_{k}^{j}(v)+\sum_{j \in N_{i}} \alpha_{j} \sum_{k \in P^{i}} t_{k}^{j}(v)-v\left(P^{i}\right) \\
& =\alpha_{P^{i}}\left(v(N)-v\left(N_{i}\right)\right)+\alpha_{N_{i}} v\left(P^{i}\right)-v\left(P^{i}\right) \\
& =\alpha_{P^{i}}\left(v(N)-v\left(N_{i}\right)-v\left(P^{i}\right)\right),
\end{aligned}
$$

where the first equality follows by definition of $h^{\alpha}$, the fifth equality follows from (i) and (ii) of Lemma 4.5, and the last equality follows since $\alpha_{P^{i}}+\alpha_{N_{i}}=1$. 
In a similar way we derive

$$
\begin{aligned}
\sum_{k \in N_{i}} h_{k}^{\alpha}(v)-v\left(N_{i}\right) & =\sum_{k \in N_{i}} \sum_{j \in N} \alpha_{j} t_{k}^{j}(v)-v\left(N_{i}\right) \\
& =\sum_{k \in N_{i}} \sum_{j \in N_{i}} \alpha_{j} t_{k}^{j}(v)+\sum_{k \in N_{i}} \sum_{j \in P^{i}} \alpha_{j} t_{k}^{j}(v)-v\left(N_{i}\right) \\
& =\sum_{j \in N_{i}} \sum_{k \in N_{i}} \alpha_{j} t_{k}^{j}(v)+\sum_{j \in P^{i}} \sum_{k \in N_{i}} \alpha_{j} t_{k}^{j}(v)-v\left(N_{i}\right) \\
& =\sum_{j \in N_{i}} \alpha_{j} \sum_{k \in N_{i}} t_{k}^{j}(v)+\sum_{j \in P^{i}} \alpha_{j} \sum_{k \in N_{i}} t_{k}^{j}(v)-v\left(N_{i}\right) \\
& =\alpha_{N_{i}}\left(v(N)-v\left(P^{i}\right)\right)+\alpha_{P^{i}} v\left(N_{i}\right)-v\left(N_{i}\right) \\
& =\alpha_{N_{i}}\left(v(N)-v\left(P^{i}\right)-v\left(N_{i}\right)\right) .
\end{aligned}
$$

From (4.8) and (4.9) it follows that

$$
\frac{1}{\alpha_{P^{i}}}\left(\sum_{k \in P^{i}} h_{k}^{\alpha}(v)-v\left(P^{i}\right)\right)=v(N)-v\left(N_{i}\right)-v\left(P^{i}\right)=\frac{1}{\alpha_{N_{i}}}\left(\sum_{k \in N^{i}} h_{k}^{\alpha}(v)-v\left(N_{i}\right)\right),
$$

which shows that the $\alpha$-TIBS Fairness condition (4.4) is satisfied in this case.

Case 2. Consider an agent $i \in N$ such that $\alpha_{P^{i}}=0$. Then $\alpha_{N_{i}}=1$ and, since $\alpha_{j}>0$ only if $j \in N_{i}$, we have

$$
\sum_{k \in P^{i}} h_{k}^{\alpha}(v)=v\left(P^{i}\right)
$$

showing that the $\alpha$-TIBS Fairness condition (4.4) is also satisfied in this case.

Case 3. Consider an agent $i \in N$ such that $\alpha_{N_{i}}=0$. Then, similar as case 2, $\alpha_{P^{i}}=1$ and, since $\alpha_{j}>0$ only if $j \in P^{i}$, we have

$$
\sum_{k \in N_{i}} h_{k}^{\alpha}(v)=v\left(N_{i}\right)
$$

showing that the $\alpha$-TIBS Fairness condition (4.4) is also satisfied in this case.

Next we state the characterization result.

Theorem 4.7 Let $\alpha \in \mathbb{R}_{+}^{n}$ be such that $\sum_{i \in N} \alpha_{i}=1$. A solution $f$ on the class of river games $\mathcal{G}^{(N, \mathcal{U})}$ satisfies the Efficiency axiom 4.1 and the $\alpha$-TIBS Fairness axiom 4.2 if and only if it is the weighted hierarchical solution $h^{\alpha}$. 
Proof. Since any hierarchical outcome is efficient, also every weighted hierarchical solution is efficient. Further it follows from Lemma 4.6 that $h^{\alpha}$ satisfies the $\alpha$-TIBS Fairness axiom 4.2. Therefore it only remains to prove that the two axioms uniquely determine a solution. Suppose that solution $f$ satisfies the two axioms and let $v \in \mathcal{G}^{(N, \mathcal{U})}$ be a river game. Since equation (4.4) in Axiom 4.2 has to hold for every $i \neq n$, the $\alpha$-TIBS fairness yields $n-1$ linear independent equations. Together with the Efficiency condition that $\sum_{i \in N} f_{i}(v)=v(N)$, we thus have $n$ linear independent equations in the $n$ unknown payoffs $f_{i}(v), i \in N$. Hence the payoffs are uniquely determined and thus it must hold that $f(v)=h^{\alpha}(v)$, for every $v \in \mathcal{G}^{(N, \mathcal{U})}$.

We conclude this subsection with considering the core-stability of weighted hierarchical solutions. In case of one spring it has been shown by Ambec and Sprumont that under Assumption 3.1 the river game is convex and thus every marginal vector of the game $v$ belongs to the core of the game. Hence in this case every hierarchical outcome is in the core and thus every weighted hierarchical solution, assigning a weighted hierarchical outcome $h^{\alpha}(v)$ to every basic river game $v$, is core-stable. Although, in contrast to the river game with one spring, the river game with multiple springs does not need to be convex, it is superadditive because $v(S)$ is the objective value of the underlying maximization problem. It also holds for every river game with multiple springs $v \in \mathcal{G}^{(N, \mathcal{U})}$ that $v(S \cup T)=v(S)+v(T)$ when $S, T \subset N, S \cap T=\emptyset$ and $S \cup T$ is not connected. Then, as has been shown by Demange (2004) within the framework of games on cycle-free graph structures, the core of game $v$ is not empty and every hierarchical outcome satisfies the core lower bounds. Since the core is a convex set, it follows that also every weighted hierarchical outcome is in the core of the game. This yields the following corollary.

Corollary 4.8 Under Assumption 3.1, every weighted hierarchical solution is core-stable on the class of river games $\mathcal{G}^{(N, \mathcal{U})}$.

\subsection{Average hierarchical solution}

When we take $\alpha_{n}=1$, and thus $\alpha_{i}=0$ for every $i \neq n$, then $h^{\alpha}(v)=t^{n}(v)$. Since $j \in P^{n}$ for every $j \neq n$ and $N_{n}=\emptyset$, the payoffs of this outcome as given in formula (4.6) reduce to

$$
t_{k}^{n}(v)=v\left(P^{k}\right)-v\left(P^{k} \backslash\{k\}\right), k \in N .
$$

In case of a basic river game with one spring this is the downstream incremental solution as proposed by Ambec and Sprumont. In its general form for games on the class $\mathcal{G}^{(N, \mathcal{U})}$ of river games with (possibly) multiple springs, it is the unique solution characterized by efficiency and the corresponding $\alpha$-TIBS fairness, which in this specific case of $\alpha_{n}=1$ requires that 
the gain in welfare that is created by joining the two coalitions $P^{i}$ and $N_{i}, i \in N$, should be fully allocated to coalition $N_{i}$. In other words, joining the coalitions $P^{i}$ and $N_{i}$ has no effect on the average utility of the agents in the upstream coalition $P^{i}$. According to Corollary 4.8 the generalized downstream incremental solution satisfies the core lower bounds for every river game with multiple springs. In fact, it is straightforward to verify that also in this case it is the unique solution that satisfies the core lower bounds and the Ambec-Sprumont aspiration level upper bounds. The downstream incremental solution for river games with multiple springs was also proposed in Khmelnitskaya (2009) as the unique solution satisfying component efficiency and another property, called successor equivalence, which generalizes the so-called upper equivalence property introduced in van den Brink, van der Laan and Vasil'ev (2007) for line-graph games.

As for the one spring case, this generalized downstream incremental solution for rivers with multiple springs has the disadvantage that all profits of cooperation between an upstream and downstream coalition goes to the agents in the downstream coalition, while the agents in the upstream coalition control the water flows from upstream to downstream. However, in this situation its counterpart, the incremental upstream solution, is not of any help. In case of one spring, the upstream incremental solution is the weighted hierarchical solution with $\alpha_{1}=1$, thus assigning all weight to the most upstream agent 1 . However, in case of multiple springs there is not a unique most upstream agent and therefore there is not a unique straightforward generalization of the upstream incremental solution. One possibility could be to take the average of all hierarchical outcomes corresponding to the agents located on one of the springs, so to take the average over all $t^{j}(v), j \in O$. Instead we consider the special case of considering the average of all hierarchical outcomes, thus the hierarchical solution corresponding to $\alpha_{i}=\frac{1}{n}$ for all $i \in N$. This average hierarchical solution, where all agents have equal weight, is characterized by Efficiency and the Equal Weights TIBS Fairness axiom and might be a good alternative to the extreme downstream incremental solution. ${ }^{12}$ In the following, we denote the average hierarchical solution by $h^{A}$.

There are several reasons to argue that the average hierarchical solution $h^{A}$ might be a good alternative for the downstream and upstream (if any) incremental solutions. First of all, according to Corollary 4.8 it is core-stable and so for every river game without externalities $v$ the core lower bounds reflecting the ATS principle are satisfied by the outcome $h^{A}(v)$. Second, consider the following. For $S \subseteq N$, let $h^{A}(S)$ denote the total payoff of the average hierarchical outcome to the agents in $S$. When, for some $i \neq n$, the upstream coalition $P^{i}$ is going to cooperate with its complement $N_{i}$, then the the equal

\footnotetext{
${ }^{12}$ Within the context of games on cycle-free graph structures this solution is introduced in Herings, van der Laan and Talman (2008) as the Average Tree solution and characterized by the so-called Component Efficiency and Component Fairness axioms. A minor adjustment of these two axioms to the river games setting gives the Efficiency and Equal Weights TIBS Fairness axioms.
} 
weights TIBS fairness property implies that

$$
\frac{1}{\left|P^{i}\right|}\left(h^{A}\left(P^{i}\right)-v\left(P^{i}\right)\right)=\frac{1}{\left|N_{i}\right|}\left(h^{A}\left(N_{i}\right)-v\left(N_{i}\right)\right) .
$$

Thus, the welfare distribution according to the average hierarchical solution has the property that for every $i \neq n$, the welfare gain of the cooperation of the upstream coalition $P^{i}$ and its complement coalition $N_{i}$ is split among the coalition $P^{i}$ and $N_{i}$ proportional to the number of agents in these two coalitions, and thus the average welfare gain of an agent in $P^{i}$ is equal to the average welfare gain of an agent in $N_{i}$.

As a special case we consider the implications of this for basic river games $v \in \mathcal{G}_{b}^{N}$. Recalling that the agents are indexed from upstream to downstream and for $j \geq i,[i, j]$ denotes the coalition of consecutive agents $i, i+1, \ldots, j$ the equal weights TIBS fairness property implies that the average weighted hierarchical outcome satisfies for every $k \neq n$

$$
\frac{1}{k}\left(h^{A}([1, k])-v([1, k])\right)=\frac{1}{n-k}\left(h^{A}([k+1, n])-v([k+1, n])\right) .
$$

Thus, the welfare distribution according to the average hierarchical solution has the property that for every $k \neq n$, the welfare gain of the cooperation of the upstream coalition $[1, k]$ and the downstream coalition $[k+1, n]$ is split among the coalition $[1, k]$ and $[k+1, n]$ proportional to the number of agents in these two coalitions, and thus the average welfare gain of an agent in $[1, k]$ is equal to the average welfare gain of an agent in $[k+1, n]$. This indeed respects the TIBS principle that, for each $k$, every agent in the coalition $[k, n]$ is entitled to a share in the water inflow at agent $k$. For the two agent case it states that the welfare gain of the cooperation between the upstream agent 1 and the downstream agent 2 is equally distributed between the two agents. It should be noticed that the equal weights TIBS fairness also holds on every subgame. In particular, for some $k$, let $v^{k}$ be the subgame on the upstream set of agents $[1, k]$, i.e., this is the $k$-player TU-game on the set $[1, k]$ when this set does not cooperate with the downstream coalition $[k+1, n]$. Further, let $A^{k}([i, j])$ denote the total payoff to the agents in the set $[i, j], 1 \leq i \leq j \leq k$, when applying the average hierarchical solution on the $k$-player TU-game $v^{k}$. Then the equal weights TIBS fairness property says, for instance, that

$$
\frac{1}{k-1}\left(A^{k}([1, k-1])-v([1, k-1])\right)=A^{k}([k, k])-v([k, k]),
$$

i.e., when agent $k$ is going to cooperate with its upstream coalition $[1, k-1]$, then the welfare gain to $k$ is a fraction $\frac{1}{k-1}$ of the total welfare gain of the agents in $[1, k-1]$. Analogously, for some $k$, let $v_{k}$ be the subgame on the downstream set of agents $[k, n]$, i.e., this is the $n-k+1$-player TU-game on the set $[k, n]$ when this set does not cooperate with the upstream coalition $[1, k-1]$. Further, let $A_{k}([i, j])$ be the total payoff to the agents 
in the set $[i, j], k \leq i \leq j \leq n$, when applying the average hierarchical solution on the TU-game $v_{k}$. Then the equal weights TIBS fairness property says, for instance, that

$$
A_{k}([k, k])-v([k, k])=\frac{1}{n-k}\left(A_{k}([k+1, n])-v([k+1, n]),\right.
$$

i.e., when agent $k$ is going to cooperate with its downstream coalition $[k+1, n]$, then the welfare gain to $k$ is a fraction $\frac{1}{n-k}$ of the total welfare gain of the agents in $[k+1, n]$. More general, when either agent $i-1$ or agent $i+k+1$ joins the coalition $[i, i+k]$ of $k$ consecutive

agents, then this agent gets a 'fair' share $\frac{1}{k+1}$ of the total welfare gain that results from joining the coalition. In this sense the average hierarchical solution on the class of basic river games meets the symmetric form of the TIBS principle as formulated by Kilgour and Dinar (1995).

\section{$5 \quad$ Weighted hierarchical solutions for river games with externalities}

In this section we consider the application of weighted hierarchical solutions to the class $\mathcal{P} \mathcal{G}^{(N, \mathcal{U})}$ of partition function form river games with externalities. Similar as without externalities, we speak about an efficient solution if it always allocates the worth of the grand coalition $N$ when all agents cooperate, thus the worth $v(N)=v(N,\{N\})$ with $v(N)$ the solution of maximization problem 3.3 for $S=N$.

\section{Axiom 5.1 Efficiency for river games with externalities}

A solution $f$ on the class of river games with externalities $\mathcal{P} \mathcal{G}^{(N, \mathcal{U})}$ is efficient if it holds for any game $v \in \mathcal{P} \mathcal{G}^{(N, \mathcal{U})}$ that $\sum_{i \in N} f_{i}(v)=v(N,\{N\})$.

Also the TIBS principle can be applied to river games with externalities in a similar way as for the no-externality case. Let $\mathcal{P}(i)$ denote the coalition structure $\left\{P^{i}, N_{i}\right\}$ and recall that the TIBS fairness axiom for games without externalities was obtained by considering the situation that the agents along the river are cooperating according to the coalition structure $\mathcal{P}(i)$ for some agent $i \neq n$, i.e., agent $i$ is cooperating with all its upstream agents in coalition $P^{i}$, while all other agents are cooperating in its complement coalition $N_{i}$. Under externalities the agents in $P^{i}$ can obtain total welfare $v\left(P^{i}, \mathcal{P}(i)\right)$ and the agents in $N_{i}$ earn $v\left(N_{i}, \mathcal{P}(i)\right)$. However, recall from Theorem 3.5 that these worths of coalitions of type $P^{i}$ and $N_{i}$ are externality-free and thus $v\left(P^{i}, \mathcal{P}(i)\right)=v_{*}\left(P^{i}\right)$, respectively $v\left(N_{i}, \mathcal{P}(i)\right)=v_{*}\left(N_{i}\right)$, being the non-cooperative core lower bounds of these coalitions. This gives us the next $\alpha$-TIBS fairness axiom for the class of river games with externalities. 


\section{Axiom 5.2 $\alpha$-TIBS Fairness for river games with externalities}

Let $\alpha \in \mathbb{R}_{+}^{n}$ be such that $\sum_{i \in N} \alpha_{i}=1$. An efficient solution $f$ on the class of river games with externalities $\mathcal{P} \mathcal{G}^{(N, \mathcal{U})}$ satisfies $\alpha$-TIBS fairness if, for any $v \in \mathcal{P G}^{(N, \mathcal{U})}$ and any $i \in N \backslash\{n\}$, it holds that

$$
\alpha_{N_{i}}\left(\sum_{k \in P^{i}} f_{k}(v)-v_{*}\left(P^{i}\right)\right)=\alpha_{P^{i}}\left(\sum_{k \in N_{i}} f_{k}(v)-v_{*}\left(N_{i}\right)\right) .
$$

Notice that this is the same as for the no externality case, only the worths of the coalitions $P^{i}$ and $N_{i}$ are replaced by their non-cooperative core lower bound values in the partition function form game $v$. So, irrespective of externalities, this axiom states that the gain in welfare that is created by joining the two coalitions $P^{i}$ and $N_{i}$, should be divided among the two coalitions proportional to the sum of the weights in these two coalitions.

Similar as in the proof of Theorem 4.7 it follows that there is a unique solution that satisfies efficiency and $\alpha$-TIBS fairness for $\alpha \in \mathbb{R}_{+}^{n}$ with $\sum_{i \in N} \alpha_{i}=1$. Moreover, similar as in the proof of Lemma 4.6 it follows straightforwardly that the weighted hierarchical solution to the associated non-cooperative lower bound TU-game $v_{*}$ satisfies both axioms. This shows the following theorem.

Theorem 5.3 Let $\alpha \in \mathbb{R}_{+}^{n}$ be such that $\sum_{i \in N} \alpha_{i}=1$. A solution $f$ on the class of river games with externalities $\mathcal{P} \mathcal{G}^{(N, \mathcal{U})}$ satisfies Efficiency and $\alpha$-TIBS Fairness if and only if $f(v)=h^{\alpha}\left(v_{*}\right)$ for every $v \in \mathcal{P} \mathcal{G}^{(N, \mathcal{U})}$.

As before, also on the class of river games with multiple springs and externalities we refer to solutions as characterized in this theorem as weighted hierarchical solutions. It assigns for a given weight vector $\alpha$ with components adding up to one, the corresponding weighted hierarchical outcome of the associated TU-game $v_{*}$.

We next show that every weighted hierarchical solution is externality free.

Definition 5.4 A solution $f$ on the class of river games with externalities $\mathcal{P G}^{(N, \mathcal{U})}$ is externality-free if the payoffs only depend on the worths of the externality-free coalitions.

We first consider the hierarchical outcome $t^{i}\left(v_{*}\right)$ in case the river has only one spring. Then formula (4.6) reduces to

$$
t_{k}^{i}\left(v_{*}\right)= \begin{cases}v_{*}([1, k])-v_{*}([1, k-1]) & \text { if } k<i, \\ v_{*}(N)-v_{*}([1, k-1])-v_{*}([k+1, n]) & \text { if } k=i, \\ v_{*}([k, n])-v_{*}([k+1, n]) & \text { if } k>i\end{cases}
$$

An agent upstream of agent $i$ receives its marginal contribution to the coalition of agents consisting of this agent and all agents upstream to it. An agent downstream of agent $i$ 
receives its marginal contribution to the coalition of agents consisting of this agent and all agents downstream to it. Finally, agent $i$ receives its marginal contribution to the grand coalition $N$, i.e., agent $i$ receives the benefit of cooperation that is obtained by connecting the upstream coalition $[1, i-1]$ and the downstream coalition $[i+1, n]$. Further, formula (5.11) shows that in every hierarchical outcome the payoffs are fully determined by the worths $v(S)$ with $S$ of either type $[1, j]$ or type $[j, n]$ for some $1 \leq j \leq n$, i.e., the payoffs are fully determined by the worths of the upstream coalitions $[1, j]$ and the downstream coalitions $[j, n], j=1, \ldots, n$. The worths of all other coalitions $[i, j], 1<i<j<n$ of consecutive agents don't affect the payoffs. Noticing that, for $i<n, P^{i}=[1, i]$ and $N_{i}=[i+1, n]$ it follows from Theorem 3.5 that every coalition that appears in the formula above is externality-free, illustrating that in case of a river with only one spring, the hierarchical outcome only depends on the worths of the externality-free coalitions and so every weighted hierarchical solution is externality-free for rivers with only one spring.

We now consider the case of multiple springs. For some $i$, first consider an agent $k \in N \backslash Q_{i}$. Then, according to formula (4.6) the payoff to $k$ in $t^{i}\left(v_{*}\right)$ is given by

$$
t_{k}^{i}\left(v_{*}\right)=v_{*}\left(P^{k}\right)-v_{*}\left(P^{k} \backslash\{k\}\right) .
$$

Since $v_{*}\left(P^{k} \backslash\{k\}\right)=\sum_{j \in U^{k}} v_{*}\left(P^{j}\right)$ it follows that the payoff $t_{k}^{i}\left(v_{*}\right)$ only depends on the worths of coalitions of type $P^{j}, j \in N$. Second, consider an agent $k \in Q_{i} \backslash\{i\}$. Then, according to formula (4.6) the payoff to $k$ in $t^{i}\left(v_{*}\right)$ is given by

$$
t_{k}^{i}\left(v_{*}\right)=v_{*}\left(N_{k^{i}}\right)-v_{*}\left(N_{k^{i}} \backslash\{k\}\right) .
$$

Inspecting $v_{*}\left(N_{k^{i}} \backslash\{k\}\right)$ it follows that $v_{*}\left(N_{k^{i}} \backslash\{k\}\right)=v_{*}\left(N_{k}\right)+\sum_{h \in U^{k} \backslash\left\{k^{i}\right\}} v_{*}\left(P^{h}\right)$. Hence, every term in the expression above is either of type $v_{*}\left(P^{i}\right)$ for some $i \in N$ or of type $v_{*}\left(N_{i}\right)$ for some $i \in N$. Apparently, also the payoff to player $i$ only depends on the worths of coalitions of type $P^{j}$ and $N_{j}, j \in N$. Since, according to Theorem 3.5 every coalition of these types is externality-free, it follows that every hierarchical outcome $t^{i}\left(v_{*}\right)$ only depends on the worths of the externality-free coalitions. This gives the following two corollaries.

Corollary 5.5 On the class $\mathcal{P} \mathcal{G}^{(N, \mathcal{U})}$ of river games with externalities, every weighted hierarchical solution $h^{\alpha}$ assigning payoff $h^{\alpha}\left(v_{*}\right)$ for every $v \in \mathcal{P} \mathcal{G}^{(N, \mathcal{U})}$ is externality-free.

Corollary 5.6 On the class $\mathcal{P} \mathcal{G}^{(N, \mathcal{U})}$ of river games with externalities, the axioms of Effciency and $\alpha$-TIBS Fairness imply externality-freeness.

We now consider core stability. Every weighted hierarchical outcome is a convex combination of the hierarchical outcomes $t^{i}\left(v_{*}\right), i \in N$. As seen before, for a river game $v$ without externalities, every hierarchical outcome is in the core of the game $v$. However, 
as we have seen in Section 3.3, for two connected, disjoint coalitions $S$ and $T$ the worth $v_{*}(S \cup T)$ can be bigger than the sum of the two worths $v_{*}(S)$ and $v_{*}(T)$. Thus, a hierarchical outcome $t^{i}\left(v_{*}\right)$ does not need to satisfy the non-cooperative core lower bound $v_{*}(R)$ for every coalition $R$. However, in Ambec and Ehlers (2008) it is argued that in river games it is natural to restrict blocking for connected coalitions, because coordination among agents becomes difficult when the agents are not neighboring. Clearly, every hierarchical outcome satisfies the non-cooperative core lower bound for every connected coalition $R$. Hence, we have the following corollary.

Corollary 5.7 For a river game with externalities $v \in \mathcal{P G}^{(N, \mathcal{U})}$, a weighted hierarchical solution satisfies the non-cooperative core lower bounds when blocking is restricted to connected coalitions.

It should be noticed that it is shown in Ambec and Ehlers (2008) that for the river game with a single spring and externalities, the downstream incremental solution (i.e. the hierarchical outcome $\left.t^{n}\left(v_{*}\right)\right)$ satisfies all non-cooperative core lower bounds. It is an open question whether this also holds for river games with multiple springs.

Finally we would like to mention that also on the class of river games with multiple springs and externalities the average hierarchical solution satisfies Equal Weights TIBSfairness. Hence, when an agent $k$ stops cooperating with its unique downstream neighbor such that coalition $P^{k}$ consisting of $k$ and all its upstream neighbors does not want to cooperate with its complement $N_{k}$, then the total payoff to the agents in $P^{k}$ is equal to $v_{*}\left(P^{k}\right)$ and the total payoff of the agents in $N_{k}$ is equal to $v_{*}\left(N_{k}\right)$. When the two coalitions cooperate and distribute the total payoff according to the average hierarchical solution, also in the game with externalities the average welfare gain of the agents in $P_{k}$ is equal to the average welfare gain of the agents in $N_{k}$.

\section{Concluding remarks}

In this paper we applied weighted hierarchical solutions, in particular the average hierarchical solution, to river games with and without externalities. These solutions give alternatives to the downstream incremental solution and upstream incremental solution. In fact, the downstream incremental solution and the upstream incremental solution (for rivers with one spring) are extreme cases of the class of weighted hierarchical solutions. We considered rivers with multiple springs and allowed for satiable agents, yielding externalities when non-connected coalitions cooperate. Using axioms underlying these solutions, in particular efficiency and fairness axioms, we argued that these principles (and corresponding solutions) are a better implementation of the TIBS principle in Kilgour and Dinar (1995) 
than the UTI principle (and corresponding downstream incremental solution) of Ambec and Sprumont (2002) which is an extreme interpretation of this principle.

For river games with one spring and nonsatiable agents the downstream incremental solution was already criticized in van den Brink, van der Laan and Vasil'ev (2007) who already proposed two alternatives. One alternative can be seen as an upstream incremental solution, being the marginal vector where agents enter from downstream to upstream, and thus assigns the dividends of cooperation fully to the upstream agents. Of course, this solution is as extreme as the downstream incremental solution but awards upstream agents for their dominant position on the river. Inspired by the equal gain split rule for sequencing problems, as a compromise van den Brink, van der Laan and Vasil'ev (2007) proposed the average of the downstream and upstream incremental solutions, and characterized it by component efficiency and an alternative fairness property stating that when two neighboring agents on the river stop cooperation, then the two 'new' river components loose the same amount of payoff. In fact, this solution is the weighted hierarchical solution with $\alpha_{1}=\alpha_{n}=\frac{1}{2}$ and $\alpha_{i}=0$ for $i \in\{2, \ldots, n-1\}$.

\section{References}

Albizuri, M.J. (2010) Games with externalities: games in coalition configuration function form, Mathematichal Methods of Operations Research, DOI 10.1007/s00186-101-03145217 (online).

Albizuri, M., Arin, J., and J. Rubio (2005), An axiom system for a value for games in partition function form, International Game Theory Review, 72, 7-63.

Ambec, S., and L. Ehlers (2008), Sharing a river among satiable agents, Games and Economic Behavior 64, 35-50.

Ambec, S., and Y. Sprumont (2002), Sharing a river, Journal of Economic Theory 107, 453-462.

Béal, S., E. Rémila, and P. Solal (2010), Rooted-tree solutions for tree games, European Journal of Operations Research 203, 404-408.

Béal, S., E. Rémila, and P. Solal (2009), Weighted component fairness for forest games, MPRA Paper No. 17455.

Bennett, L., S. Ragland, and P. Yolles (1998), Facilitating agreements through an interconnected game approach: the case of river basin, in R. Just and S. Netanyahu 
(eds.) Conflict and Cooperation in Transboundary Water Resources, Kluwer Publishing Company, Norwell, MA.

Bondareva, O. (1963), Some applications of linear programming methods to the theory of cooperative games, Problemy Kybernetiki 10, 119-139 (in Russian).

Brink, R. van den, G. van der Laan, and V.A. Vasil'ev (2007), Component efficient solutions in line-graph games with applications, Economic Theory 33, 349-364.

Carraro, C., C. Machiori, and A. Sgobbi (2005a), Applications of negotiation theory to water issues, World Bank Policy Working Paper 3641, World Bank, Washington.

Carraro, C., C. Machiori, and A. Sgobbi (2005b), Advances in negotiation theory: bargaining, coalitions and fairness, World Bank Policy Working Paper 3642, World Bank, Washington.

Clippel, G. de, and R. Serrano (2008), Marginal contributions and externalities in the value, Econometrica 76, 1413-1436.

Demange, G. (2004), On group stability in hierarchies and networks, Journal of Political Economy 112, 754-778.

Dinar, A., A. Ratner, and D. Yaron (1992), Evaluating cooperative game theory in water resources, Theory and Decision 32, 1-20.

Dinar, A., K. Kemper, W. Blomquist, M Diez, G Sine, and W. Fru (2005), Decentralization of river basin management: a global analysis, World Bank Policy Working Paper 3637, World Bank, Washington.

Funaki, Y., and T. Yamato (1999), The core of an economy with a common pool resource: a partition function form approach, International Journal of Game Theory 28, 157171.

Gillies, D.B. (1953), Some theorems on n-person games, Princeton University Press, Princeton, NJ.

Gomez, A. (2005), Multilateral contracting with externalities, Econometrica 73, 13291350 .

Gomez, A., and P. Jehiel (2005), Dynamic processes of social and economic interactions: on the persistence of inefficiencies, Journal of Political Economy 113, 626-667.

Herings, P.J.J., G. van der Laan, and A.J.J. Talman (2007), The socially stable core in structured transferable utility games, Games and Economic Behavior 59, 85-104. 
Herings, P.J.J., G. van der Laan, and A.J.J. Talman (2008), The average tree solution for cycle-free graph games, Games and Economic Behavior 62, 77-92.

Khmelnitskaya, A.B. (2009), Values for rooted-tree and sink-tree digraph games and sharing a river, Theory and Decision, DOI 10.1007/s11238-009-9141-7.

Kilgour, D.M., and A. Dinar (1995), Are stable agreements for sharing international river water now possible?, Policy Research Working Paper 1474, World Bank, Washington.

Kilgour, D.M., and A. Dinar (2001), Flexible water sharing within an international river basin, Environmental and Resource Economics 18, 43-60.

Meade, J.E. (1973), The theory of economic externalities: The control of environmental pollution and similar social costs, Sijhoff, Leiden.

Ray, D., and R. Vohra (1999), A theory of endogenous coalition structures, Games and Economic Behavior 26, 286-336.

Shapley, L. S. (1953), A value for N-person games, in: Contributions to the Theory of Games, Vol II (eds. H. W. Kuhn, and A. W. Tucker), Princeton University Press, Princeton, 307-317.

Shapley, L.S. (1967), On balanced sets and cores, Naval Research Logistics Quarterly 14, 453-460.

Supalla, R., B. Klaus, O. Yeboah, and R. Bruins (2002), A game theory approach to deciding who will supply instream flow water, Journal of the American Water Resources Association 38, 959-966.

Thrall, R., and W. Lucas (1963), n-person games in partition function form, Naval Research Logistic Quarterly 10, 281-298. 